\title{
AUFSÄTZE
}

\section{Parlament und Verwaltung: in Rollenpartnerschaft vereint? Ein Plädoyer für die Verknüpfung von Parlaments- und Verwaltungsforschung}

\author{
Sabine Kropp und Matthias Ruschke
}

Das Verhältnis von Verwaltung und Politik zählt zu den Kernthemen verwaltungspolitologischer Studien. ${ }^{1}$ Erst kürzlich hat sich ein Sonderheft der Politischen Vierteljahresschrift (PVS) wieder explizit diesem Thema gewidmet. ${ }^{2}$ Grenzt man den Untersuchungsgegenstand auf die Interaktionen von Parlament und Verwaltung ein, erkennt man jedoch auffallende Forschungslücken. Die Gründe hierfür liegen nicht zuletzt in der zunehmenden Spezialisierung und Professionalisierung der Politikwissenschaft als wissenschaftlicher Disziplin. Beide Zweige, die Parlaments- wie die politikwissenschaftliche Verwaltungsforschung, bewegen sich in weitgehend voneinander isolierten Zirkeln; personelle und inhaltliche Verschränkungen gibt es kaum. Von der Sache her sind beide Forschungsfelder jedoch eng miteinander verbunden. Gerade in parlamentarischen Regierungssystemen, die in den Mitgliedstaaten der EU dominieren, stellt die Gewaltenverschränkung ein prägendes Element dar. Die Regierung hängt vom Vertrauen der Parlamentsmehrheit ab; die Politikformulierung erfolgt in Arbeitsparlamenten, zu denen auch der Deutsche Bundestag zählt, in enger Kooperation zwischen Regierung, Ministerialverwaltung und Abgeordneten. ${ }^{3}$ Effektives Regieren setzt voraus, dass diese Staatsleitung „auch als eine gemeinsame Aufgabe von Politik und Verwaltung wahrgenommen, akzeptiert und praktiziert wird“ “ . Regierung und Verwaltung sind demzufolge wichtige Rollenpartner der Abgeordneten; dies gilt insbesondere für die Abgeordneten der die Regierung tragenden Mehrheitsfraktionen. ${ }^{5}$

1 Vgl. Jörg Bogumil/ Werner Jann, Verwaltung und Verwaltungswissenschaft in Deutschland. Einführung in die Verwaltungswissenschaft, Wiesbaden 2009, S. $186 \mathrm{ff}$.

2 Vgl. dies. I Frank Nullmeier (Hrsg.), Politik und Verwaltung, PVS Sonderheft 37, Wiesbaden 2006.

3 Vgl. Friedrich Bischoff / Michael Bischoff, Parlament und Ministerialverwaltung, in: Hans-Peter Schneider / Wolfgang Zeh (Hrsg.), Parlamentsrecht und Parlamentspraxis in der Bundesrepublik Deutschland. Ein Handbuch, Berlin 1989, S. 1457 - 1477, S. 1457 ff.; vgl. Sabine Kropp, Ausbruch aus „exekutiver Führerschaft"? Ressourcen- und Machtverschiebungen im Dreieck von Regierung, Verwaltung und Parlament, in: Jörg Bogumil / Werner Jann / Frank Nullmeier (Hrsg.), a.a.O. (Fn. 2), S. $275-298$, S. 290.

4 Werner J. Patzelt, Verwaltungskultur in der Ministerialbürokratie: Ein empirischer Essay, in: Everhard Holtmann / Werner J. Patzelt (Hrsg.), Führen Regierungen tatsächlich? Zur Praxis gouvernementalen Handelns, Wiesbaden 2008, S. 115 - 130, S. 116 ff. Vgl. Günther Schmid / Hubert Treiber, Bürokratie und Politik. Zur Struktur und Funktion der Ministerialbürokratie in der Bundesrepublik Deutschland, München 1975; vgl. Rolf-Richard Grauhan, Modelle politischer Verwaltungsführung, in: PVS, 10. Jg. (1969), H. 2, S. $269-284$.

5 Vgl. Werner J. Patzelt, The Constituency Roles of MPs at the Federal and Länder Levels in Germany, in: Regional and Federal Studies, 17. Jg. (2007), H. 1, S. 47 - 70, S. 62. 
Verwaltungspolitologische Untersuchungen haben Interaktionen von Politik und Verwaltung in der Vergangenheit mitunter als eine Art Nullsummenspiel betrachtet: Im Mittelpunkt stand die Frage, wer von beiden führt und wer geführt wird. ${ }^{6}$ Die Bürokratie wird dabei als der dominierende Part angesehen. ${ }^{7}$ Jedoch hängt die reale Machtverteilung nicht zuletzt von der bestehenden Akteurskonstellation und den Strategien der Akteure ab. Treffsichere Einschätzungen werden außerdem dadurch erschwert, dass viele Kooperationen in informellen Bahnen und damit jenseits der formalen Institutionen und des direkt Wahrnehmbaren ablaufen und empirisch mühsam zu erheben sind. ${ }^{8}$ Es liegen somit keineswegs erschöpfende Antworten auf die Frage vor, inwiefern Abgeordnete zum Beispiel auf informellem Wege Funktions- und Informationsverluste - etwa über den direkten Kontakt zur Ministerialbürokratie - kompensieren können. ${ }^{9}$

Die Europäisierung nationalstaatlicher Entscheidungsprozesse hat zweifelsohne dazu geführt, dass die Parlamente gegenüber den Exekutiven einen Steuerungs- und Machtverlust erlitten haben. Nationale Abgeordnete finden auf EU-Ebene keine in den europäischen Politikzyklus institutionell eingebettete Möglichkeit der Mitentscheidung. Die Regierungen, aber auch die in die Arbeitsgruppen der EU-Kommission, des EU-Ministerrates, in die Komitologie sowie in die nationalstaatliche Vorbereitung von Entscheidungen der EUInstitutionen eingebundenen nationalen Ministerialbürokratien gelten daher als die eigentlichen Gewinner der Europäisierung. ${ }^{10}$ Die These der „Entparlamentarisierung “ verweist damit, obwohl sie inzwischen deutlich differenzierter betrachtet wird ${ }^{11}$ und mitunter bereits von einer "Reparlamentarisierung" die Rede ist, nicht zuletzt auf die machtumverteilenden Effekte sowie auf die Abkopplung der Parlamente von Entscheidungsprozessen im Mehrebenensystem der EU. Die Fachpolitiker der Fraktionen sind weniger als die Beamten in der Lage, nationale und europäische Arenen miteinander zu verknüpfen. Es ist somit davon auszugehen, dass die Europäisierung beinahe aller Politikfelder auch auf das Rollenverständnis von Abgeordneten und Ministerialbürokratie zurückwirkt, da sie zum Beispiel den Expertenstatus beider Gruppen unmittelbar berührt.

Es gibt folglich gute Gründe, zentrale Fragestellungen der Parlaments- und Verwaltungsforschung enger als bisher zusammenzuführen. Dabei gewinnt vor allem die Frage nach

6 Vgl. Everhard Holtmann / Werner J. Patzelt (Hrsg.), a.a.O. (Fn. 4); dies. (Hrsg.), Kampf der Gewalten? Parlamentarische Regierungskontrolle - gouvernementale Parlamentskontrolle. Theorie und Empirie, Wiesbaden 2004.

7 Vgl. Carl Böhret, Politik und Verwaltung. Beiträge zur Verwaltungspolitologie, Opladen 1983, S. 132 ff.; ders., Politik und Verwaltung. Verwaltungspolitik als Suche nach einem ausgeglichenerem Verhältnis von Politik und Verwaltung, in: Hans-Georg Wehling (Hrsg.), Verwaltung und Politik in der Bundesrepublik, Stuttgart 1986, S. 36 - 53.

8 Vgl. Friedrich Bischoff / Michael Bischoff, a.a.O. (Fn. 3); Christian Hanke, Informale Regeln als Substrat des parlamentarischen Verhandlungssystems. Zur Begründung einer zentralen Kategorie der Parlamentarismusforschung, in: ZParl, 25. Jg. (1994), H. 3, S. 410 - 440.

9 Vgl. aber Manfred Schwarzmeier, Parlamentarische Mitsteuerung, Wiesbaden 2001, S. 221 ff.; Everhard Holtmann, Die öffentliche Verwaltung, in: Oscar W. Gabriel / ders. (Hrsg.), Handbuch des politischen Systems Deutschlands, München 2005, S. 333 - 371, S. 348.

10 Vgl. exemplarisch für die Niederlande mit empirischen Befunden Karin Geuijen / Paul t'Hart / Sebastiaan Princen / Kutsal Yesilkagit, The New Eurocrats. National Civil Servants in EU PolicyMaking, Amsterdam 2008.

11 Vgl. für viele John O'Brennan / Tapio Raunio, National Parliaments within the Enlarged European Union. From Victims of Integration to Competitive Actors, London 2007. 
den - möglicherweise gewandelten - Rollenorientierungen von Politikern und Beamten an Bedeutung. Das eigene Rollenverständnis sowie die Einschätzung des jeweiligen Interaktionspartners legen Handlungsoptionen und Strategien wesentlich fest; Rollenbilder gelten als zuverlässige Prädiktoren für Verhalten. ${ }^{12}$ Sind Rollenteilungen unklar oder gehen die Akteure von falschen Erwartungen an ihr jeweiliges Gegenüber aus, sind Störungen oder gar Blockaden im Regierungsprozess vorprogrammiert. Vertrauen hingegen ist ein unverzichtbares „Schmiermittel“ im Getriebe des Regierens. ${ }^{13}$ Ein tragfähiges „Intra-Elite-Vertrauen" bildet sich voraussichtlich je eher aus, desto mehr das Verhalten der Beteiligten mit den wechselseitig antizipierten Rollenerwartungen übereinstimmt und Selbst- wie Fremdwahrnehmungen deckungsgleich sind.

Dieser Beitrag arbeitet den Forschungsstand zum oben umrissenen Thema im Wesentlichen für den deutschen Kontext mit dem Ziel auf, bestehende Forschungslücken genauer auszuleuchten und Hypothesen für weitere Untersuchungen zu generieren. Er versteht sich als nachdrückliches Plädoyer für eine engere Verknüpfung von Parlaments- und Verwaltungsforschung. Angesichts der Vielfalt an forschungsrelevanten Aspekten, die sich unter dem weit gefassten Thema „Parlament und Verwaltung" subsumieren lassen, konzentrieren sich die folgenden Ausführungen auf einen Ausschnitt, der für die Steuerungsfähigkeit von Politik essentiell ist: die Rollenverständnisse von Abgeordneten und Ministerialbeamten und deren wechselseitige Zuordnung. Es sind letztlich solche Perzeptionen, die handlungsleitend sind und Interaktionen prägen, nicht jedoch allein die Selbstwahrnehmung der Akteursgruppen. ${ }^{14}$ Weichen die vom Gegenüber zugewiesenen Rollen deutlich vom jeweils eigenen Selbstverständnis ab, sind Missverständnisse vorprogrammiert: Wo klaffen Fremdund Selbstwahrnehmung also auseinander? Sind die Rollenverständnisse beider Seiten nicht kompatibel, dürfte dies zudem ein nicht unerhebliches Erschwernis für effektives Regieren darstellen: Definieren Abgeordnete und Ministerialbürokratie ihre Rollen tatsächlich auf ähnliche Weise? Falls nicht: Wird dies als funktionale Verschiedenartigkeit - also als sinnvolle Rollenteilung - akzeptiert oder als Problem betrachtet?

Angesichts des weitreichenden Europäisierungsgrads in vielen Politikfeldern stellt sich ferner die Frage, inwieweit sich Rollen, die überwiegend auf den Aktionsraum nationaler Politik bezogen sind, verändern, wenn Abgeordnete und Beamte die europäische Arena strategisch in ihr Handeln einzubeziehen beginnen. Während zum ersten Teilaspekt, dem jeweils eigenen Rollenverständnis von Parlament und Verwaltung, durchaus aufschlussreiche Forschungsergebnisse vorliegen, muss der Forschungsstand zum zweiten Aspekt, den wechselseitigen Rollenzuschreibungen, als lückenhaft bezeichnet werden (vgl. Tabelle 1). Der dritte Gesichtspunkt, die Europäisierung von Rollenverständnissen, ist ebenfalls noch weitgehend unerforscht. Da die Europäisierung in vielen Politikfeldern jedoch weit vorangeschritten ist, gibt es keine überzeugenden Gründe, diesen Gesichtspunkt in Unter-

12 Vgl. Hans-Ulrich Derlien / Renate Mayntz, Bundeselite 1970-1987. Rollenverständnis und Werthaltungen im Wandel, Bamberg 1991, S. 2 ff.

13 Vgl. William D. Leach / Paul A. Sabatier, To Trust an Adversary: Integrating Rational and Psychological Models of Collaborative Policymaking, in: American Political Science Review, 99. Jg. (2005), H. 4, S. $491-503$.

14 Vgl. für viele Fritz W. Scharpf, Games Real Actors Play. Actor-Centered Institutionalism in Policy Research, Boulder 1997. 
suchungen länger auszublenden. Der Steuerungs- und Implementationszusammenhang von Politik erstreckt sich im europäisierten Bundesstaat schließlich über mehrere Ebenen.

\section{Rollenverständnis der Verwaltung und ibre Interaktionen mit Politik und Parlament - Ergebnisse der Verwaltungsforschung}

Wie interagiert die Verwaltung mit der Politik, und welche Rollen schreiben sich Beamte dabei selbst und den Abgeordneten zu? Im Laufe jahrzehntelanger Forschungen haben sich vier normative Leitbilder der öffentlichen Verwaltung herausgeschält, die diese Wechselbeziehung spiegeln: die „autonome Verwaltung“, die „hierarchische“, die „kooperative“ sowie die „responsive Verwaltung“. ${ }^{15}$ Das Leitbild der „kooperativen Verwaltung“ kann als das derzeit vorherrschende Paradigma gelten. Es sieht die Bürokratie nicht nur als kompetenten Verhandlungspartner in Politiknetzwerken, sondern auch als deren Architekten. In diesem Leitbild ist die idealtypische Unterscheidung von Politik und Verwaltung teilweise aufgehoben. ${ }^{16}$ Die Gegenüberstellung von „klassischem“ und „politischem“ Beamten erfasst die Realität längst nur noch ausschnitthaft: Der klassische Beamte Max Webers verfügt idealtypisch über ein monistisches Verständnis von Staatsinteressen und ist von der Richtigkeit objektiver Standards überzeugt. Probleme können aus seiner Sicht sachlich und objektiv gelöst werden; über den Parteizwist fühlt er sich erhaben. Der politische Beamte hingegen erkennt die pluralistische Verfasstheit des öffentlichen Interesses an und bejaht die Legitimität unterschiedlicher Gruppeninteressen. Er betrachtet Politiker als Teilnehmer an einem gemeinsamen „Spiel“ ${ }^{17}$ Während der klassische Beamte somit eher regel- und prozedural orientiert ist, verhält sich der politische Beamte stärker programm- oder problemorientiert. ${ }^{18}$

Die oben skizzierten Typisierungen von Verwaltung dienten empirischen Untersuchungen als konzeptionelle Grundlage, um das Rollenverständnis der Bürokratie zu erfassen. Schon die Studie von Bärbel Steinkemper stellte einen Rückgang des klassischen Bürokraten und zugleich eine zunehmende Politisierung der Bürokratie fest. Eine Differenzierung zwischen Politik und Verwaltung sei, zumindest in Spitzenpositionen, nicht mehr möglich, da die Verwaltung wegen der Politisierung ihrer Führungskräfte selbst „politischen“ Charakter angenommen habe. ${ }^{19}$ Alle nachfolgenden Studien bestätigten im Großen und Ganzen diese Durchbrechung idealtypischer Rollen von Bürokratie und Politik. ${ }^{20}$

15 Vgl. Werner J. Patzelt, a.a.O. (Fn. 4), S. $124 \mathrm{ff}$

16 Vgl. Bärbel Steinkemper, Klassische und politische Bürokraten in der Ministerialverwaltung der Bundesrepublik Deutschland. Eine Darstellung sozialstruktureller Merkmale unter dem Aspekt politischer Funktionen der Verwaltung, Köln 1974; Robert D. Putnam, Die politischen Einstellungen der Ministerialbeamten in Westeuropa - Ein vorläufiger Bericht, in: PVS, 17. Jg. (1976), H. 1, S. $23-61$.

17 Vgl. Robert D. Putnam, a.a.O. (Fn. 16), S. $25 \mathrm{ff}$.

18 Vgl. Werner Jann, Politik als Aufgabe der Bürokratie: Die Ministerialbürokratie im politischen System der Bundesrepublik im Vergleich zu anderen westlichen Demokratien, in: Politische Bildung, 21. Jg. (1988), H. 2, S. $39-56$, S. 52 ff.

19 Vgl. Bärbel Steinkemper, a.a.O. (Fn. 16), S. 62.

20 Vgl. Hans-Ulrich Derlien, Verwaltung zwischen Berufsbeamtentum und Parteipolitik: Personalrekrutierung und Personalpatronage im öffentlichen Dienst, in: Politische Bildung, 21. Jg. (1988), H. 2, S. 57 - 72; ders., Mandarins or Managers? The Bureaucratic Elite in Bonn - 1970 to 1987 and Beyond, in: Governance, 16. Jg. (2003), H. 3, S. $401-428$. 
Die Studie von Aberbach, Putnam und Rockman ${ }^{21}$ - ein Klassiker der vergleichenden Verwaltungsforschung - ging über diese Dichotomie hinaus, indem sie vier unterschiedliche Rollenbilder konstruierte. Der erste Typus entspricht dem des „klassischen Bürokraten“ im Sinne Webers, also einem Bürokraten mit einer unpolitischen und ausführenden Rolle. Der zweite beschreibt den „kritischen Bürokraten“, der sich als weitgehend interessenfreier Experte versteht. Der dritte Typus kann als „politisierter Bürokrat“ beschrieben werden. Dieser tritt als Experte und Mediator zwischen eng definierten, organisierten Interessen auf. Als vierter schließlich wurde der „hybride Bürokrat“ benannt, der hinsichtlich seines Rollenverständnisses kaum noch von einem Politiker zu unterscheiden ist. ${ }^{22}$ Aberbach u.a. gingen in ihrer Studie davon aus, dass sich die administrativen Führungskräfte immer mehr in Richtung des hybriden Bürokraten entwickeln und sich letztlich vom weberianischen Rollenbild verabschieden würden.

Um diese Rollenbilder empirisch ermitteln zu können, schufen die Autoren verschiedene Rollenaspekte, so den „technician“, „advocate“, „legalist“, „broker“, „trustee“, „facilitator“, den „partisan“, „policymaker“ oder „ombudsman“, und fügten diese zu Rollen zusammen. ${ }^{23}$ Diese wurden wiederum mit dem Rollenverständnis der Abgeordneten verglichen. Auch wechselseitige Einschätzungen beider Gruppen wurden erfragt. ${ }^{24}$ Ein zentrales Ergebnis der Studie lautete, dass die klassische Rollenteilung zwischen Politikern und Bürokraten in der Tat überholt sei. Insbesondere die Phase der Politikformulierung ist von einer engen Zusammenarbeit zwischen beiden Seiten geprägt, wobei sich Politiker und Bürokraten als gleichberechtigte Partner verstehen. Beide fühlen sich als „Policymaker“. Dennoch unterscheiden sie sich hinsichtlich ihres Problemverständnisses und ihrer bevorzugten Problemlösungsstrategien. Bürokraten nähern sich Problemen eher mit einem „technischen“ Vorverständnis ihrer Aufgabe und bevorzugen präzise Lösungen für klar definierte Aufgaben. Politiker beurteilen Probleme hingegen stärker unter dem Gesichtspunkt der politischen Durchsetzbarkeit, der Anwaltschaft für bestimmte Gruppen und der Wiederwahl. Eine umfassende Konvergenz beider Gruppen konnte nicht bestätigt werden, vielmehr ließ sich das Selbstverständnis der Ministerialbürokratie als Mischform des kritischen und politisierten Bürokraten beschreiben. Die Studie von Aberbach u.a. übte seit ihrem Erscheinen einen prägenden Einfluss auf die vergleichende Verwaltungsforschung aus und ist, trotz mancher Kritik, bis heute Anstoß zahlreicher Untersuchungen. ${ }^{25}$

Hans-Ulrich Derlien und Renate Mayntz ${ }^{26}$ hatten den deutschen Teil der internationalen Vergleichsstudie von 1987 übernommen. Ihnen zufolge ist die Beamtenrolle in ihrer Selbst-

21 Vgl. Joel D. Aberbach / Robert D. Putnam / Bert A. Rockman, Bureaucrats and Politicians in Western Democracies, Cambridge 1981.

22 Vgl. ebenda, S. 4 ff.

23 Vgl. ebenda, S. $86 \mathrm{ff}$.

24 Vgl. ebenda, S. $102 \mathrm{ff}$.

25 Vgl. zusammenfassend Kwang-Hoon Lee / Jos C.N. Raadschelders, Political-Administrative Relations: Impact of and Puzzles in Aberbach, Putnam, and Rockman 1981, in: Governance, 21. Jg. (2008), H. 3, S. 419 - 438; Werner Jann, Praktische Fragen und theoretische Antworten: 50 Jahre Policy-Analyse und Verwaltungsforschung, in: PVS, 50. Jg. (2009), H. 3, S. 475 - 505, S. 483.

26 Vgl. Hans-Ulrich Derlien / Renate Mayntz, Comparative Elite Study II. Einstellungen der politisch-administrativen Elite des Bundes 1987, Bamberg 1988; dies., Party Patronage and Politicization of the West German Administrative Elite 1970-1987 - Towards Hybridization?, in: Governance, 2. Jg. (1989), H. 4, S. 384 - 404; dies, Bundeselite 1970-1987 - Rollenverständnis und Werthaltungen im Wandel, Bamberg 1991. 
wie in der Fremdwahrnehmung maßgeblich von Expertenwissen geprägt. ${ }^{27}$ 45,9 Prozent der Beamten glaubten seinerzeit, Beamte verfügten über mehr Fachwissen als Politiker; allerdings teilten diese Meinung nur 28,8 Prozent der Politiker. ${ }^{28}$ Beide Gruppen wiesen in ihren Karrierewegen zudem Ähnlichkeiten, aber auch Unterschiede auf: Sie zeigten eine gewisse Affinität zum öffentlichen Dienst, die bei den Beamten aber stärker ausgeprägt war. ${ }^{29}$ Auch diese Studie bestätigte, dass der „politisierte“ Beamte der vorherrschende Typus sei $^{30}$, eine weitere „Hybridisierung“ der Rollen habe seit 1970 nicht stattgefunden. ${ }^{31} \mathrm{Je}$ höher die Position innerhalb der Verwaltungshierarchie, desto eher werden politische Aspekte als bedeutsam eingeschätzt und desto größer ist die Wahrscheinlichkeit einer Parteimitgliedschaft. ${ }^{32}$ In Studien, die nach der deutschen Einheit entstanden, wurden zudem regionale Unterschiede festgestellt. In den ostdeutschen Bundesländern dominierte in den 1990er Jahren zum Beispiel der Typus des „klassischen Beamten“ stärker als im Westen. ${ }^{33}$ Inwieweit es hier zu einer allmählichen Angleichung gekommen ist oder ob sich nicht doch pfadabhängige Entwicklungen ausbilden, ist eine ebenfalls noch unbeantwortete Frage.

Eine abermalige Zunahme autonomen Verwaltungshandelns wäre mit dem viel zitierten Übergang zum „neo-weberianischen Staat“ verbunden, in dem eine Transformation der traditionell-hierarchischen Steuerung hin zur dezentralen Anreiz- und Leistungsorientierung des Managerialismus vollzogen wird. Ein Kernelement des so genannten New Public Management (NPM) ist die stärkere Trennung von Politik und Verwaltung. ${ }^{34}$ Eine derarti-

27 Vgl. dies., Bundeselite 1970-1987, a.a.O. (Fn. 26), S. 327.

28 Vgl. dies., Comparative Elite Study II, a.a.O. (Fn. 26), S. 12.

29 Vgl. dies., Bundeselite 1970-1987, a.a.O. (Fn. 26), S. 67 ff.

30 Vgl. dies., Comparative Elite Study II, a.a.O. (Fn. 26), S. 39 f.; dies., Party Patronage and Politicization of the West German Administrative Elite 1970-1987, a.a.O. (Fn. 26); siehe auch die Elitenstudie von 1995 und 1981, vgl. Kai-Uwe Schnapp, Soziodemographische Merkmale der bundesdeutschen Elite, in: Wilhelm Bürklin / Hilke Rebenstorf (Hrsg.), Eliten in Deutschland, Rekrutierung und Integration, Opladen 1997, S. 101 - 122, S. 115 f.; Ursula Hoffmann-Lange, Eliten, Macht und Konflikt in der Bundesrepublik, Opladen 1992, S. 158 ff. Zu Karriereverläufen vgl. auch Hans-Ulrich Derlien, Die politische und die administrative Elite der Bundesrepublik, in: Werner Jann / Klaus König (Hrsg.), Regieren zu Beginn des 21. Jahrhunderts, Tübingen 2008, S. 291 - 328, S. 318.

31 Vgl. Hans-Ulrich Derlien / Renate Mayntz, Party Patronage and Politicization of the West German Administrative Elite 1970-1987, a.a.O. (Fn 26), S. 395 ff.

32 Vgl. Joel D. Aberbach / Hans-Ulrich Derlien / Renate Mayntz / Bert A. Rockman, American and German Federal Executives - Technocratic and Political Attitudes, in: International Social Science Journal, 42. Jg. (1990), H. 1, S. 3 - 18, S. 12 f.; Hans-Ulrich Derlien, Soziale Herkunft und Parteibindung der Beamtenschaft. Ein Beitrag zum Politisierungsproblem, in: Hans-Georg Wehling (Hrsg.), a.a.O. (Fn. 7), S. 115 - 132, S. 127 f.

33 Vgl. Horst Damskis / Bärbel Möller, Verwaltungskultur in den neuen Bundesländern. Werte und Einstellungen von Führungskräften in den Ministerialverwaltungen von Brandenburg und Sachsen, Frankfurt am Main 1997; Horst Damskis, Regionale Verwaltungskulturen in Ostdeutschland. Politik- und Rollenverständnis von leitenden Ministerialbeamten in Brandenburg und Sachsen, Dissertation Humboldt-Universität Berlin, Berlin 1996; Christoph Reichard / Eckhard Schröter, Rollenverhalten und Einstellungen von Führungskräften in der (Ost- und West-)Berliner Verwaltung, in: Wolfgang Seibel / Arthur Benz / Heinrich Mäding (Hrsg.), Verwaltungsreform und Verwaltungspolitik im Prozeß der deutschen Einigung, Baden-Baden 1993, S. 207 - 217, S. 209 f.

34 Vgl. Geert Bouckeart, Auf dem Weg zu einer neo-weberianischen Verwaltung. New Public Management im internationalen Vergleich, in: Jörg Bogumil / Werner Jann / Frank Nullmeier (Hrsg.), a.a.O. (Fn. 2), S. $345-372$. 
ge, an ökonomischen Anreizen ausgerichtete Steuerung ginge, wenn sie von der Verwaltung als handlungsleitendes Prinzip verinnerlicht wird, auch mit veränderten Rollenorientierungen einher. Inwieweit sich ein solcher Wandel tatsächlich vollzogen hat, kann auf der Grundlage empirischer Studien bislang nur ansatzweise beantwortet werden. Deutschland gilt im internationalen Vergleich jedenfalls als eher vorsichtiger Modernisierer. ${ }^{35}$ Studien zur kommunalen Ebene, die allerdings nur wenige Rückschlüsse auf das Verhältnis zwischen Parlament und Ministerialbürokratie auf Bundes- und Landesebene zulassen, weisen darauf hin, dass im Verwaltungsalltag der Rückgriff auf altbewährte Muster dominiert. ${ }^{36}$ Jedoch hat sich der Bund bei der Einführung von Elementen des neuen Steuerungsmodells ohnehin nicht als Vorreiter profiliert, so dass eine umfassende Neudefinition von Verwaltungsrollen beziehungsweise ein echter Kulturwandel zu einem managerialistischen Verwaltungsmodell wohl eher nicht zu erwarten ist. Zwar können gewisse Effekte dieser inzwischen jahrzehntelangen Diskurse auf das Rollenbild der Verwaltung nicht von vornherein ausgeschlossen werden ${ }^{37}$ : Erste Ergebnisse, die zwischen dem Selbstverständnis von Beamten in Ministerien und weniger unmittelbar der politischen Spitze unterstellten Agenturen unterscheiden, weisen aber darauf hin, dass die bloße Erweiterung von Managementautonomie nicht automatisch zu einer geringeren Politisierung führt. Vielmehr hängt diese von der politischen Bedeutung des Tätigkeitsbereichs ab. ${ }^{38}$

Rollenorientierungen der Verwaltungselite wurden 2005 und zuletzt 2009 in einer umfassenden Studie untersucht. ${ }^{39}$ Diese replizierte die Arbeiten von Aberbach u.a. sowie von Derlien / Mayntz und konzentrierte sich auf das Rollenverständnis der Verwaltungselite. Welche Rollen die Abgeordneten der Verwaltung zuschreiben und umgekehrt und ob das Rollenverständnis beider Seiten kompatibel ist, stand aber nicht im Mittelpunkt. Auch in dieser Untersuchung wurden nur die Verwaltungsspitzen erfasst; sie ging somit nur bedingt

35 Vgl. für viele Christopher Pollit / Geert Bouckeart, Public Management Reforms: A Comparative Analysis, Oxford 2004.

36 Vgl. Jörg Bogumil / Stephan Grohs / Sabine Kuhlmann, Ergebnisse und Wirkungen kommunaler Verwaltungsmodernisierung in Deutschland - eine Evaluation nach zehn Jahren Praxiserfahrung, in: Jörg Bogumil / Werner Jann / Frank Nullmeier (Hrsg.), a.a.O. (Fn. 2), S. 151 - 184; zusammenfassend Jörg Bogumil / Werner Jann, a.a.O. (Fn. 1), S. 248 f.

37 Vgl. hierzu den so genannten „diskursiven Institutionalismus“, Vivien A. Schmidt, Discursive Institutionalism: The Explanatory Power of Ideas and Discourse, in: Annual Review of Political Science, 11. Jg. (2008), S. $303-326$.

38 Vgl. Falk Ebinger / Carina Schmitt, Alles eine Frage des Managements? Wie Autonomierechte die Handlungsfreiheit des administrativen Führungspersonals beeinflussen, in: PVS, 51. Jg. (2010), H. 1, S. 69 - 93. Eine Studie über die Niederlande fand heraus, dass eine größere formale Autonomie von Agencies keineswegs mit einer tatsächlichen politischen Unabhängigkeit einhergeht, vgl. Kutsal Yesilkagit, Political Influence and Bureaucratic Autonomy, in: Public Organization Review, 8. Jg. (2008), H. 2, S. 137 - 153; vgl. auch B. Guy Peters / John Pierre, Politization of the Civil Service in Comparative Perspective: The Quest for Control, London 2004; vgl. zudem Katja Schwanke / Falk Ebinger, Politisierung und Rollenverständnis der deutschen administrativen Elite 1970 bis 2005 - Wandel trotz Kontinuität, in: Jörg Bogumil / Werner Jann / Frank Nullmeier (Hrsg.), a.a.O. (Fn. 2), S. $228-249$.

39 Vgl. Katja Schwanke / Falk Ebinger, a.a.O. (Fn. 38); Falk Ebinger / Linda Jochheim, Wessen loyale Diener? Wie die Große Koalition die deutsche Ministerialbürokratie veränderte, in: der moderne staat - Zeitschrift für Public Policy, Recht und Management, 3. Jg. (2009), H. 2, S. 335 - 353. 
auf Unterschiede ein, die innerhalb der Verwaltungshierarchie gegeben sein dürften. ${ }^{40}$ Dass die Führungselite grundsätzlich eine größere Affinität gegenüber der Politik aufweist als etwa die Referatsebene, liegt jedoch nahe. Gleichwohl liefert die Studie erhellende Indizien dafür, wie sich Rollenorientierungen der Verwaltungselite über die Zeit entwickelt haben; sie bietet daher die Grundlage für einen diachronen Vergleich.

Erneut hoben die leitenden Beamten in den Bundesministerien hervor, politischen Aspekten komme für ihre Arbeit eine herausragende Bedeutung zu. $\mathrm{Zu}$ unterscheiden ist dabei zwischen einer formalen Politisierung der Beamten, die von der Mitgliedschaft in einer Partei geprägt ist, und einer funktionalen, derzufolge politische Aspekte von der Verwaltung berücksichtigt werden. ${ }^{41}$ Erstere war 2005 noch immer stark ausgeprägt: 75 Prozent der Staatssekretäre, 60 Prozent der Abteilungsleiter und ein Drittel der Unterabteilungsleiter waren im Untersuchungszeitraum Parteimitglieder. Während der rot-grünen Regierungszeit, die zum ersten Mal in der Geschichte der Bundesrepublik einen kompletten Austausch der Regierung mit sich brachte, stieg diese Zahl bei der Neubesetzung von Positionen sogar weiter an. ${ }^{42} \mathrm{Im}$ Gegensatz zu den älteren Befragungen schätzen sich die Führungskräfte heute aber selbst als weniger politisiert ein. ${ }^{43} \mathrm{Ob}$ dieses Antwortverhalten in den allgemeinen gesellschaftlichen Trend wachsenden Parteienverdrusses eingeordnet werden kann, bleibt einstweilen ungeklärt. Fragt man, wie sich die Beamten den oben bereits beschriebenen Idealtypen zuordnen, so ermittelt man nahezu die gleichen Werte wie die Vorgängerstudien und damit ein recht stabiles Selbstverständnis. Beamte betrachten sich in erster Linie als Experten mit Fachwissen, als Umsetzer politischer Vorgaben, als Repräsentanten des Staates, als Initiatoren politischer Projekte sowie als Vermittler zwischen konfligierenden Interessen. Sie bewerteten 2005 die politische Seite ihrer Arbeit mit rund 60 Prozent als sehr positiv - ein Wert, der im Vergleich zu früher gesunken ist und 2009 noch niedriger ausfiel. ${ }^{44}$ Beinahe alle Befragten gaben an, dass sie im Verwaltungsalltag - im Sinne einer funktionalen Politisierung - zwischen fachlichen und politischen Aspekten abwägen; dieser Wert stieg während der Großen Koalition von 2005 bis 2009 noch an. Dabei zeigten sich rund zwei Drittel ihrer politischen Funktion sehr bewusst. ${ }^{45}$ Ebenfalls über zwei Drittel der Befragten gaben an, dass sich ihr eigenes Berufsbild mit dem der Politiker, das heißt auch der Abgeordneten, teilweise überlappe - gegenüber 1970 eine Zunahme von 45 Prozent. Eine große Überschneidung mit der Rolle von Politikern kann aber nicht nachgewiesen werden. Eine stärkere Hybridisierung könnte aber die wachsende Zahl derjenigen anzeigen, die eine Unterstützung der bisherigen Ressortpolitik selbst dann befürworten, wenn sie im Widerspruch zu einem neuen Kurs nach einem Regierungswechsel steht. Die Bindung an eigene fachliche und politische Überzeugungen verdrängt offenbar zunehmend das Rollenverständnis des loyalen, der rechtmäßigen Regierung verbundenen Bürokraten

40 Ähnlich auch Werner J. Patzelt, Verwaltungskultur in der Ministerialbürokratie: Ein empirischer Essay, a.a.O. (Fn. 4).

41 Vgl. Katja Schwanke / Falk Ebinger, a.a.O. (Fn. 38), S. 234 ff.

42 Ein Vergleich mit 2009, der eine Zunahme der Parteilosen erbrachte, ist dennoch schwierig, weil sich offenkundig das Antwortverhalten der Befragten verändert hat. Vgl. Falk Ebinger / Linda Jochheim, a.a.O. (Fn. 39), S. $334 \mathrm{ff}$.

43 Vgl. Katja Schwanke / Falk Ebinger, a.a.O. (Fn. 38), S. 242.

44 Vgl. ebenda, S. 243; Falk Ebinger / Linda Jochheim, a.a.O. (Fn. 39), S. 339; Hans-Ulrich Derlien / Renate Mayntz, Comparative Elite Study II, a.a.O. (Fn. 26).

45 Vgl. im Folgenden Katja Schwanke / Falk Ebinger, a.a.O. (Fn. 38), S. 244 f. 
Weber'schen Typs. ${ }^{46}$ Einschränkend sei jedoch hinzugefügt, dass es sich bei den Befragten ausschließlich um Führungskräfte der öffentlichen Verwaltung handelt, die der Politik grundsätzlich näher stehen als die Beamten auf niedrigeren Hierarchieebenen.

Wie nehmen sich Beamte und Politiker beziehungsweise Abgeordnete wechselseitig wahr? Aus Verwaltungsstudien lassen sich, wie gezeigt, nur einige wenige, inzwischen schon ältere Befunde entnehmen. ${ }^{47}$ Geht es um die Einschätzung der politischen Rolle und den Expertenstatus der Beamten, fallen Fremd- und Selbstwahrnehmung offenbar auseinander. Während 76,9 Prozent der Beamten angaben, die politische Seite ihrer Arbeit gefalle ihnen sehr gut, bewerteten nur 25,4 Prozent der Abgeordneten beziehungsweise Politiker diese „politische“ Seite der Arbeit von Beamten ebenfalls positiv. ${ }^{48}$

Elitestudien bestätigten im Großen und Ganzen den Befund, dass die wechselseitigen Rollenwahrnehmungen unterschiedlich ausfallen. Verwaltungs- und politische Elite schätzen sich zwar in etwa als gleich stark ein, wenn es um die Durchsetzung ihrer eigenen Interessen geht. ${ }^{49}$ Die Verwaltungselite wird durch die Gesamtelite jedoch als kompetenter als die politische Elite bewertet. Gleichzeitig vergibt sie selbst aber für die politische Elite die besten Werte, was wiederum darauf hinweist, dass durch enge Kooperation und angesichts der Zugehörigkeit zum politisch-administrativen System und, damit einhergehend, nicht unähnlicher Rollenverständnisse der Gegenseite durchaus Kompetenzen bescheinigt werden. Die Verwaltungseliten wiederum werden von den Eliten des politischen Sektors differenziert beurteilt: Die Politiker, die dem Regierungslager angehören, betrachten die Ministerialbürokratie als deutlich kompetenter als die der Opposition. ${ }^{50}$ Dieser Befund vermag insofern nicht zu überraschen, als die Kooperationsbeziehungen zwischen regierungstragenden Fraktionen und Ministerialbürokratie im System des Neuen Dualismus erheblich enger sind als jene zwischen dieser und den Oppositionsfraktionen. In den Arbeitskreisen beziehungsweise Arbeitsgruppen der Fraktionen, die dem Regierungslager angehören, sind zum Beispiel häufig auch die thematisch jeweils zuständigen Beamten anwesend. Zudem werden Staatssekretäre und Ministerialdirektoren, wie eine jüngere verwaltungssoziologische Studie erneut bestätigt ${ }^{51}$, nach Regierungswechseln in großem Umfang ausgewechselt. Sie stehen daher dem Regierungslager nahe, was das Vertrauen der Opposition in die Verwaltung und die Kompetenzzuschreibungen nicht erhöht. Dass die Sympathie der Abteilungs- und Unterabteilungsleiter in den Ministerien auch 2005 mehrheitlich noch eher der Opposition als der Regierung galt, weist darauf hin, dass die Unterabteilungsleiter häufig noch in der Ära Helmuth Kohls rekrutiert worden waren. ${ }^{52}$ Allerdings bemüht sich die Ministerialbürokratie in der Regel selbst um ein gutes Verhältnis zur Opposition, da sie ihre Vorhaben auch über

46 Vgl. Falk Ebinger / Linda Jochheim, a.a.O. (Fn. 39), S. 338.

47 Vgl. Joel D. Aberbach / Robert D. Putnam / Bert A. Rockman, a.a.O. (Fn. 21), S. $102 \mathrm{ff}$.

48 Vgl. Hans-Ulrich Derlien / Renate Mayntz, Comparative Elite Study II, a.a.O. (Fn. 26), S. 10.

49 Vgl. im Folgenden Martina Sauer, Durchsetzungsfähigkeit und Kooperationspotential von Eliten als Bausteine der Elitenintegration, in: Wilhelm Bürklin / Hilke Rebenstorf (Hrsg.), Eliten in Deutschland, Rekrutierung und Integration, Opladen 1997, S. 285 - 320.

50 Vgl. dies., Gesellschaftliche Steuerungschancen durch Elitenintegration. Kommunikation und Kooperation bundesdeutscher Funktionsträger vor dem Hintergrund funktionaler Differenzierung, Opladen 2000, S. $260 \mathrm{ff}$.

51 Vgl. Hans-Ulrich Derlien, a.a.O. (Fn. 20), S. 317; Katja Schwanke / Falk Ebinger, a.a.O. (Fn. 38), S. 241.

52 Vgl. Falk Ebinger / Carina Schmitt, a.a.O. (Fn. 38). 
einen Regierungswechsel hinweg absichern möchte. Vollständig „abgehängt“ vom administrativen Betrieb sind somit auch die Oppositionsfraktionen nicht. ${ }^{53}$

Dass zwischen Politik und anderen Sektoren stabile Kommunikationsnetzwerke bestehen, bestätigte auch die Potsdamer Elitestudie. Das politisch-administrative System und seine Akteure, also Regierung, Verwaltung und Parlament, befinden sich im Zentrum der Steuerung und Kommunikation. ${ }^{54}$ Der Verwaltungselite kommt dabei eine "Scharnierfunktion" zu: Sie weist intensive Kontakte zum Parlament auf, vor allem zu den Bundestagsausschüssen ${ }^{55}$, deren Zuschnitt die fachliche Zuständigkeit der Ministerien spiegelt. Ministerialbürokratie und Parlament verhalten sich zueinander wie kommunizierende Röhren $^{56}$ : Dass Beamte sich selbst eine politische Rolle zuschreiben, erleichtert Interaktionen mit dem Parlament. Die Struktur des Arbeitsparlaments mit seinen Fachausschüssen und den spezialisierten Arbeitskreisen der Fraktionen stellt dabei eine institutionelle Voraussetzung für die enge Vernetzung beider Seiten dar.

Fasst man den Kern dieser Verwaltungs- und Elitestudien zusammen, lassen sich folgende Ergebnisse festhalten: Alle Untersuchungen bestätigen, dass sich die Führungskräfte der Ministerialbürokratie heute nachdrücklich als in den politischen Prozess eingebettet verstehen. Sie sehen sich als Experten und Rechtsanwender, schätzen die politische Seite ihrer Arbeit, weniger aber die Politisierung von außen. Als reine Rechtstechniker begreifen sie sich nicht. Angesichts enger Vernetzungen im Politikbetrieb stufen sie ihr parlamentarisches Gegenüber, also die Abgeordneten, als kompetenter ein, als dies andere Teileliten tun. Ihre Rollen sind durchweg „parlamentskompatibel“. Die Organisation des parlamentarischen Betriebes und die Strukturen des Arbeitsparlaments in Deutschland fördern und stabilisieren die Affinität der Verwaltung zur Politik - und umgekehrt. Dass die Opposition die Verwaltung dabei als weniger kompetent betrachtet, als dies die Regierungsfraktionen tun, korrespondiert wiederum mit der formalen Politisierung der Verwaltungsspitze.

Als Forschungsdesiderat muss insbesondere der Rollenquervergleich zwischen Verwaltung und Parlament gelten. Dieser ist in den neueren Studien kaum mehr enthalten; lediglich den älteren Untersuchungen lassen sich Anhaltspunkte entnehmen. Nur spärliche Hinweise gibt es zudem auf die (möglichen) Effekte der jahrzehntelangen Debatte über Verwaltungsmodernisierung auf das Selbstverständnis der Beamten. Wie sich die offenbar abnehmende Loyalität des administrativen Spitzenpersonals gegenüber der Politik auf das Intra-Elite-Vertrauen und die Steuerungsfähigkeit der Politik auswirkt, bedarf ebenfalls weiterer Untersuchungen.

53 Vgl. Michael Traeger, Bildungspolitik in der Bundesrepublik Deutschland. Eine Länder vergleichende Netzwerkanalyse anhand ausgewählter Kooperationsbeispiele in Sachsen-Anhalt und Rheinland-Pfalz, Marburg 2005.

54 Vgl. Martina Sauer, a.a.O. (Fn. 49), S. 73 ff; siehe auch Martina Sauer / Kai-Uwe Schnapp, Elitenintegration durch Kommunikation? Eine Analyse der Kontaktmuster der Positionseliten, in: Wilhelm Bürklin / Hilke Rebenstorf(Hrsg.), Eliten in Deutschland. Rekrutierung und Integration. Opladen 1997, S. 239 - 284, S. 264.

55 Vgl. Martina Sauer, Gesellschaftliche Steuerungschancen durch Elitenintegration? Kommunikation und Kooperation bundesdeutscher Funktionsträger vor dem Hintergrund funktionaler Differenzierung, Opladen 2000, S. $147 \mathrm{ff}$.

56 Vgl. hierzu auch die Elitestudien von Ursula Hoffmann-Lange, Wer gehört zur Machtelite der Bundesrepublik? Ergebnisse einer Elitestudie von 1981, in: Hans-Georg Wehling (Hrsg.), Eliten in der Bundesrepublik Deutschland, Stuttgart 1990, S. 164 - 178; dies., Eliten, Macht und Konflikt in der Bundesrepublik, Opladen 1992. 


\section{Abgeordnetenrollen - mit den Rollen der Ministerialbürokratie kompatibel?}

Welche Rollen ordnen sich die Abgeordneten selbst zu, und schließt das oben dargestellte Rollenverständnis der Verwaltung an das der deutschen Abgeordneten an? Erste Aussagen hierzu lassen sich aus dem breit gefächerten Schrifttum der Parlamentsforschung herleiten. Es liegen zwar zahlreiche Studien zu Abgeordnetenrollen in Deutschland vor, jedoch unternehmen auch sie keinen expliziten Rollenvergleich mit der Verwaltung. Allerdings finden sich Hinweise über Beziehungen zwischen Parlament und Verwaltung, die, in der Zusammenschau mit Studien über die Verwaltung, die Ausarbeitung forschungsleitender Annahmen erlauben.

Die Rollentheorie ist in der deutschen Parlamentsforschung eines der am häufigsten angewandten heuristischen Instrumente. ${ }^{57}$ Wer nun aber versucht, sich einen Überblick über den Forschungsstand zu verschaffen, stößt bei erstem Betrachten auf ein Dickicht an unterschiedlichen, sich überlappenden und nur mühsam zu systematisierenden Abgeordnetenrollen. Viele Rollen, die in Studien zur vergleichenden Parlamentsforschung herausgearbeitet wurden, sind zudem durch die jeweilige institutionelle Ausgestaltung des Parlamentarismus bestimmt und lassen sich daher nicht ohne weiteres von einem nationalen Kontext auf einen anderen beziehen. Deshalb nehmen die nachfolgenden Ausführungen insbesondere auf die Arbeiten beziehungsweise auf jene Rollen Bezug, die für die Interaktionen von Politik und Verwaltung im deutschen Regierungssystem von Bedeutung sind.

Inspiriert durch frühe US-amerikanische Forschungen der 1960er Jahre, wurden wesentliche Aspekte der Rollentheorie für den deutschen Kontext adaptiert und weiter entwickelt. ${ }^{58}$ Wahlke und seine Mitautoren ${ }^{59}$ umrissen in ihrer Studie unter anderem Zweckrollen, Klientelrollen sowie allgemein geteilte Rollen und konstruierten daraus ein Netzwerk parlamentarischer Rollenorientierungen, die wechselseitig aufeinander einwirken. Als wegweisend gelten vor allem die von ihnen typisierten Repräsentationsrollen, die den Abgeordneten (unter Rückgriff auf ältere Repräsentationstheorien, zum Beispiel von Edmund Bur$k e$ ) entweder als „delegate“, das heißt als Beauftragten der Wähler, oder als „trustee“, als dem Gewissen verpflichteten Treuhänder, beschreiben, oder ihn in einer mittleren Position als „politico“ sehen. ${ }^{60}$ Ein weiteres Rollenset wurde unter anderem von James David Barber

57 Vgl. Werner J. Patzelt, Wahlkreisstil und Abgeordnetenrollen: Grundzüge eines Forschungsprogramms, in: ZParl, 20. Jg. (1989), H. 1, S. 114 - 150; siehe auch Helmar Schöne / Julia von Blumenthal, Parlamentarismus im politischen System der Bundesrepublik Deutschland. Rückblick und Ausblick nach 40 Jahren - Zur Einführung, in: dies. (Hrsg.), Parlamentarismusforschung in Deutschland. Ergebnisse und Perspektiven 40 Jahre nach Erscheinen von Gerhard Loewenbergs Standardwerk zum Deutschen Bundestag, Baden-Baden 2009, S. 9 - 33, S. 19 ff.

58 Vgl. Werner J. Patzelt, a.a.O. (Fn. 57); ders., Abgeordnete und Repräsentation. Amtsverständnis und Wahlkreisarbeit, Passau 1993; Thomas Saalfeld / Wolfgang C. Müller, Roles in Legislative Studies: A Theoretical Introduction, in: dies. (Hrsg.), Members of Parliament in Western Europe: Roles and Behaviour, London / Portland 1997, S. 1 - 16; Jürgen von Oertzen, Das Expertenparlament. Abgeordnetenrollen in den Fachstrukturen bundesdeutscher Parlamente, Baden-Baden 2006, S. $40 \mathrm{ff}$.

59 Vgl. John C. Wablke / Heinz Eulau / William Buchanan / LeRoy C. Ferguson, The Legislative System. Explorations in Legislative Behavior, New York / London 1962.

$60 \mathrm{Vgl}$. Thomas Saalfeld / Wolfgang C. Müller, a.a.O. (Fn. 58), S. 6 f.; Jürgen von Oertzen, a.a.O. (Fn. 58), S. 41. 
1965 mit den Rollen der „lawmakers“, „spectators“, „advertisers“ und „reluctants“ entwickelt. $^{61}$

Als weiterer Meilenstein gilt die auf das britische Unterhaus bezogene Studie von Donald $D$. Searing von $1994^{62}$, der neben den formalen Rollen des „parliamentary private secretary“, des „whip“, des ,junior minister“ sowie des „minister“ auch informale Rollen wie den Wahlkreisvertreter (constituency member), den Fachpolitiker (policy advocate), den Organisator (parliament man) oder den Karrierepolitiker (ministerial aspirant) identifizierte. ${ }^{63}$ Diese Überlegungen, die zwischen Positions- und Präferenzrollen unterscheiden, wurden von Studien, welche die Rollentheorie im Sinne des Rational-Choice-Paradigmas deuten, aufgegriffen und uminterpretiert. ${ }^{64}$

Über den Deutschen Bundestag ist in den vergangenen Jahren ebenfalls eine ganze Reihe von Arbeiten erschienen ${ }^{65}$, durch die das Rollenkonzept in der deutschen Parlamentsforschung weiter ausdifferenziert werden konnte. Die Abgeordneten wurden gefragt, wie sie die verschiedenen Facetten ihrer Tätigkeit einschätzen. Aus den so ermittelten Rollenmerkmalen wurden schließlich Gesamtrollen zusammengefügt: So etwa der Wahlkreisarbeiter, der sich - nach dem Schwerpunkt seiner Tätigkeit - vom Parlamentsarbeiter abgrenzen lässt. Letztere wurden wiederum in drei Subtypen unterteilt: der Fachpolitiker, der als Spezialist in Arbeitsgremien und Ausschüssen auftritt, der Abgeordnete der parlamentarischen Führungsmannschaft (Manager), der sich durch Fachwissen auszeichnet und der auf das Vertrauen seiner Fraktion beziehungsweise der parlamentarischen Organisation angewiesen ist, sowie der Abgeordnete, der angesichts der Kompatibilität von Amt und Mandat der Regierungsmannschaft angehört. Dieser beschränkt die Wahlkreisarbeit angesichts zeitlicher Belastungen und wegen der Regierungsverantwortung auf das Notwendige. Beschrieben wurden ferner der vom Parlamentsbetrieb unabhängige Abgeordnete, der sich wiederum von denjenigen abgrenzen lässt, für die das Mandat zum Beruf geworden ist; der Jungparlamentarier, der den Routiniers im Parlamentsbetrieb gegenübersteht; der „solide Arbeiter“, der als Gegentyp zum "Selbstdarsteller“ gelten kann, der wiederum von der

61 Vgl. James David Barber, The Lawmakers: Recruitment and Adaptation to Legislative Life, New Haven / London 1965.

62 Vgl. Donald D. Searing, Westminster's World. Understanding Political Roles, Cambridge 1994; zur Bedeutung der Studie vgl. Thomas Saalfeld / Wolfgang C. Müller, a.a.O. (Fn. 58), S. 9 ff.

63 Vgl. hierzu ausführlich Jürgen von Oertzen, a.a.O. (Fn. 58); vgl. ders., Rollentheorie als Werkzeug der Parlamentarismusforschung, in: Helmar Schöne / Julia von Blumenthal (Hrsg.), a.a.O. (Fn. 57), S. $217-230$.

64 Vgl. Kaare Strom, Rules, Reasons and Routines: Legislative Roles in Parliamentary Democracies, in: Wolfgang C. Müller / Thomas Saalfeld (Hrsg.), a.a.O. (Fn. 58), S. $153-174$.

65 Ausgehend von einer anfänglich explorativ angelegten Forschungskonzeption vgl. Werner J. Patzelt, Neuere Repräsentationstheorie und das Repräsentationsverständnis von Abgeordneten, in: Zeitschrift für Politik, 38. Jg. (1991), H. 2, S. 166 - 199; ders., Abgeordnete und Repräsentation, a.a.O. (Fn. 58); ders., German MPs and Their Roles, in: Wolfgang C. Müller / Thomas Saalfeld (Hrsg.), a.a.O. (Fn. 58), S. 55 - 78; ders. / Karin Algasinger, Abgehobene Abgeordnete? Die gesellschaftliche Vernetzung der deutschen Volksvertreter, in: ZParl, 32. Jg. (2001), H. 3, S. 503 - 527. Zu berufsspezifischen Einstellungen und Rollen vgl. Werner J. Patzelt, Der "ideal“" Abgeordnete. Parlamentarier beschreiben ihren Beruf, in: Politische Studien, 42. Jg. (1991), H. 3, S. 497 - 512; ders., Deutschlands Abgeordnete: Profil eines Berufsstands, der weit besser ist als sein Ruf, in: ZParl, 27. Jg. (1996), H. 3, S. 462 - 502; mit quantitativen Erhebungen vgl. ders., a.a.O. (Fn. 5). 
Ministerialbürokratie voraussichtlich weniger als Experte akzeptiert wird, sowie der Gegensatz zwischen Vollzeitparlamentarier und Teilzeitparlamentarier. ${ }^{66}$

Das Repräsentationsverständnis der Abgeordneten ist von vielen unterschiedlichen und häufig miteinander konkurrierenden Rollen geprägt: Sie sind Vertreter der Fraktion, des Volkes, sie müssen ihren Wahlkreis pflegen, sind ihrer Partei auf der lokalen wie auf der nationalen Ebene verpflichtet und durch Mehrfachmitgliedschaften an organisierte gesellschaftliche Interessen - von religiösen bis berufsständischen Gruppen - gebunden. Sie treten damit auch als Advokaten sektoraler Orientierungen auf, die vor allem dann als handlungsleitend vermutet werden können, wenn sie nicht nur mit einer einfachen Mitgliedschaft, sondern mit formalen Positionen in Interessengruppen verbunden sind. Abgeordnete bewegen sich auf unterschiedlichen Feldern, die wiederum verschiedene Handlungsrationalitäten aufweisen: Sie gehören in der Regel einer Fraktion an, sie sind Mitglieder einer Parteiorganisation, deren programmatische Ausrichtung nicht deckungsgleich mit jener der Fraktion sein muss, und sie sind Vertreter regionaler und lokaler Interessen. Strategisches Handeln setzt voraus, dass bei konkurrierenden Anforderungen Präferenzen über diese verschiedenen Arenen hinweg verrechnet werden müssen - in den Worten der Spieltheorie müssten Abgeordnete also eine „Hauptarena“ bestimmen. ${ }^{67}$ Diese Festlegung kann grundsätzlich auf unterschiedliche Weise und nach situativ variierenden Kalkülen erfolgen. Die Verkopplung dieser unterschiedlichen Arenen stellt eine besondere Herausforderung an den Abgeordnetenberuf dar. Durch sie unterscheidet sich der Alltag eines Parlamentariers von dem der Ministerialbürokratie, die einen derartigen Spagat zwischen unterschiedlichen Repräsentationsrollen nicht bewältigen muss. Dies erklärt auch, warum die Expertise der Abgeordneten durchschnittlich weniger stark ausgeprägt ist als die der Verwaltung, die sich nicht nur stärker spezialisieren kann, sondern auch weniger Responsivitätsaufgaben übernehmen muss. Beamte bleiben überdies durchschnittlich länger im Amt.

Fragt man die deutschen Abgeordneten, als wessen Vertreter sie sich verstehen, so lösen sie diese verschiedenen Anforderungen überwiegend in einem - eher diffus anmutenden Gemeinwohlbezug auf. 86 Prozent fühlten sich in einer nach der deutschen Einheit durchgeführten Umfrage in erster Linie dem Gemeinwohl verbunden. Rund zwei Drittel sahen sich als Vertreter der Bürger im Wahlkreis und 58 Prozent als Repräsentant ihrer Wähler. 34 Prozent der Abgeordneten verstanden sich als Vertreter ihrer Parteien und 13 Prozent als Anwälte nahestehender Interessengruppen. ${ }^{68}$ Dieses Repräsentationsverständnis hat sich offenbar über die Zeit hinweg nicht wesentlich verändert ${ }^{69}$ - wir haben jedoch nur be-

66 Vgl. Werner J. Patzelt, Abgeordnete und ihr Beruf. Interviews - Umfragen - Analysen, Berlin 1995, S. 257 ff.

67 Vgl. George Tsebelis, Nested Games. Rational Choice in Comparative Politics. Berkeley / Los Angeles / Oxford 1990.

68 Vgl. Werner J. Patzelt, Deutschlands Abgeordnete, a.a.O. (Fn. 65), S. 468.

69 Vgl. Heinrich Best / Michael Edinger / Stefan Jahr / Karl Schmitt, Abgeordnetenbefragung 2003/04 - Zwischenauswertung, Friedrich-Schiller Universität Jena, Sonderforschungsbereich 580, Jena 2005, http://www.sfb580.uni-jena.de/typo3/uploads/media/Deutscher_Bundestag.pdf (Abruf am 7. Juli 2009); Heinrich Best / Michael Edinger / Karl Schmitt / Lars Vogel, Zweite Deutsche Abgeordnetenbefragung 2007 - Gesamtergebnis, Friedrich-Schiller Universität Jena, Sonderforschungsbereich 580, Jena 2008, http://www.sfb580.uni-jena.de/typo3/uploads/media/Abgeordnetenbefragung_2007.pdf (Abruf am 7. Juli 2009); dies., Zweite Deutsche Abgeordnetenbefra- 
grenztes Wissen darüber, in welcher Weise es die Abwägung eines Abgeordneten zwischen konkurrierenden Anforderungen im Entscheidungsalltag prägt. Sofern die Interessen von Wahlkreis und Allgemeinwohl kollidieren, wird dieser Konflikt den Angaben von 60 Prozent der befragten Abgeordneten zufolge zugunsten des Gemeinwohls gelöst. ${ }^{70}$ Da dieses aber nicht a priori feststeht, sondern in pluralistischen Gesellschaften offen für verschiedene - auch parteipolitisch geprägte - Deutungen ist, dürfte die jeder Repräsentationsleistung innewohnende Spannung zwischen Gemeinwohl und Partikularinteressen in der Regel nicht gänzlich unlösbar sein. Interessen der Partei beziehungsweise Fraktion sowie des Wahlkreises können angesichts dieser grundsätzlichen Deutungsoffenheit als im Gemeinwohl aufgehoben betrachtet werden.

Die Abgeordneten nehmen innerhalb des Parlaments offenbar eine gewisse Arbeitsteilung vor: 35 Prozent legen den Schwerpunkt eher auf die Parlamentsarbeit, 12 Prozent mehr auf die Wahlkreisarbeit, die Mehrheit (53 Prozent) betrachtet das Verhältnis als ausgewogen. Etwa zwei Drittel der Abgeordneten bezeichnen ihre Rolle dabei eher als die eines Fachpolitikers denn eines Generalisten. Sie schreiben sich damit Kompetenz für ein oder mehrere Politikfelder zu - was aber nicht unbedingt heißen muss, dass sie sich den Ministerialbeamten in dieser Expertenrolle ebenbürtig fühlen oder von diesen auch als Fachmann akzeptiert werden. Eine explorative Studie über den Bundestag deutet in diesem Zusammenhang darauf hin, dass die „Wahlkreisarbeiter" weniger auf Interaktionen mit der Ministerialverwaltung ausgerichtet sind als jene Abgeordneten, die sich explizit als Experten auf einem Politikfeld verstehen. Die Ministerialbürokratie wird als Rollen- und Interaktionspartner offenbar unterschiedlich eingeschätzt. Einige Abgeordnete bezeichneten deren Informationsvorsprung als kaum einholbar, andere wiederum verwiesen darauf, dass ihr eigenes Fachwissen - ebenso wie ihr Steuerungswissen - dem der Ministerialbürokratie durchaus ebenbürtig sei. ${ }^{71}$ Überlegen seien die Abgeordneten den Beamten jedoch mit Blick auf ihre Repräsentationsrollen insofern, als sie die Durchsetzbarkeit von politischen Vorhaben angesichts der durch das Mandat engeren Anbindung an Wählerpräferenzen besser beurteilen könnten als die Bürokratie.

In Abgeordnetenstudien finden sich einige Anhaltspunkte, die das Verhältnis der Parlamentarier zur Ministerialbürokratie beleuchten. Den Funktionsbedingungen des neuen Dualismus entsprechend, machen insbesondere die Angehörigen des Regierungslagers von der engen Verknüpfung zwischen Exekutive und Legislative Gebrauch. 70 Prozent der Abgeordneten betrachten die Ministerien und 62 Prozent die Regierung als wichtigen Interaktionspartner und bedeutende Informationsquelle für ihre Parlamentsarbeit. Auch den nachgeordneten Ämtern, Behörden und Verwaltungen wurde dabei ein hoher Stellenwert beigemessen. ${ }^{72}$

gung 2007 - Dokumentation für den Bundestag, Friedrich-Schiller Universität Jena, Sonderforschungsbereich 580, Jena 2008, http://www.sfb580.uni-jena.de/typo3/uploads/media/ Dokumentation_Bundestag.pdf (Abruf am 7. Juli 2009).

70 Vgl. im Folgenden Werner J. Patzelt, Deutschlands Abgeordnete, a.a.O. (Fn. 65), S. 469.

71 Vgl. Sabine Kropp, German Parliamentary Party Groups in Europeanised Policymaking - Awakening from the Sleep? Institutions and Heuristics as MPs' Resources, in: German Politics, 19. Jg. (2010), H. 2, S. 123 - 147. Befragt wurden neben Abgeordneten auch Mitarbeiter der Fraktionen.

72 Vgl. Werner J. Patzelt, a.a.O. (Fn. 5), S. 62; ders., Deutschlands Abgeordnete, a.a.O. (Fn. 65), S. 478. 
Für die Wahlkreisarbeit sind insbesondere Kontakte zur kommunalen Verwaltung wichtig. ${ }^{73}$ Dies stützt die oben formulierte Annahme, dass „Wahlkreisarbeiter“ weniger die Verbindung zur Ministerialbürokratie suchen als die Fachpolitiker. In einer Studie über die Rollenorientierungen der ostdeutschen Bundestagsabgeordneten ${ }^{74}$ wurde die Ministerialbürokratie ebenfalls als wichtiger und unentbehrlicher Rollenpartner beschrieben. Es existiere eine fachliche, kollegiale und vertrauensvolle Beziehung, und zwar trotz der Kontrollfunktion, welche die Legislative gegenüber der Exekutive wahrnimmt. Die Ministerialbürokratie gilt den Abgeordneten, auch denen der Opposition, als wichtige Informationsquelle. Bei den befragten ostdeutschen Parlamentariern wurde festgestellt, dass diese im Austausch mit den Ministerien das direkte Gespräch bevorzugen; meistens wird der Kontakt unmittelbar mit der Fachebene aufgenommen. Fallen die Informationen nicht zufriedenstellend aus, wenden sich die Abgeordneten aber auch an leitende Ministerialbeamte. Eine ältere Erhebung über Jungparlamentarier ergab zudem, dass 62 Prozent „zu wenig Einfluss auf die Ministerialbürokratie" als den größten Nachteil ihrer parlamentarischen Arbeit ansahen. ${ }^{75}$ Selbst über die Hälfte der neuen Abgeordneten der Regierungspartei erachtete den zu geringen Einfluss auf die Ministerialbürokratie noch als das größte Problem. Bei der Opposition lagen diese Werte, ihrer größeren Distanz zur Exekutive entsprechend, höher. Die Altparlamentarier nannten diesen Aspekt nur an dritter Stelle, als Defizit betrachteten es aber immerhin noch 46 Prozent. Bei aller Vorsicht, die geboten ist, wenn man diese Ergebnisse in die Gegenwart projiziert und Befunde unterschiedlicher Studien miteinander verknüpft, weisen die fast dreißig Jahre alten Zahlen doch darauf hin, dass sich die Abgeordneten - ungeachtet der Expertenrolle, die sich viele selbst zuschreiben - dennoch nicht als gleichwertiger Partner der Bürokratie fühlen und dies auch als ein Steuerungsund Kontrollproblem empfinden. ${ }^{76}$ Zugleich untermauern diese Befunde die Annahme, dass das Verhältnis zwischen beiden Seiten, zumindest was die Expertenrolle betrifft, im Großen und Ganzen asymmetrisch angelegt ist.

Die hier referierten Ergebnisse zeigen, dass es im Rollenverständnis von Abgeordneten und Ministerialbürokratie Überschneidungen, aber auch Unterschiede gibt. Gemeinwohlorientierung und die nachrangige Bedeutung von partikularen Gruppeninteressen sowie das Bestreben, sachlich angemessene Lösungen zu finden, prägen das artikulierte Selbstverständnis beider Gruppen. Die Führungskräfte in den Ministerien messen der politischen Seite ihrer Arbeit hohe Bedeutung bei - angesichts der umfänglichen formalen Politisierung ist dies kein überraschender Befund. Anders als die Abgeordneten müssen die ohnedies stärker auf ein Teilgebiet spezialisierten Beamten weniger miteinander konkurrierende Rollenanforderungen vereinbaren. Dies wiederum kommt der Ausübung der Expertenrolle zugute. Ungeklärt ist bislang, welche Unterschiede sich in den Rollenverständnissen auf

73 Vgl. Werner J. Patzelt / Karin Algasinger, a.a.O. (Fn. 65), S. 506 f.

74 Vgl. im Folgenden Joachim Edward Krieger, Rollenorientierungen, Rollenerwartungen und Rollenverhalten von Ost-Abgeordneten im Deutschen Bundestag. Eine empirische Untersuchung, Frankfurt am Main 1998.

75 Ewald Rose / Joachim Hofmann-Göttig, Selbstverständnis und politische Wertungen der Bundestagsabgeordneten. Ergebnisse repräsentativer Umfragen, in: ZParl, 13. Jg. (1982), H. 1, S. 62 84, S. $67 \mathrm{ff}$.

76 Vgl. hierzu auch Dietrich Herzog / Hilke Rebenstorf / Camilla Werner / Bernhard Weßels, Abgeordnete und Bürger. Ergebnisse einer Befragung der Mitglieder des 11. Deutschen Bundestages und der Bevölkerung, Opladen 1990, S. 117 ff. 
den unterschiedlichen Hierarchiestufen der Verwaltung finden lassen und wie die verschiedenen, in den oben zitierten Studien ermittelten „Rollentypen“ von Abgeordneten die Verwaltung einschätzen. Der Literatur lassen sich zumindest Hinweise entnehmen, dass die Wahrnehmung der Verwaltung durch die Parlamentarier variiert, je nachdem, welcher Typus von Rollenträger befragt wird. Offen ist ebenfalls, ob die Selbsteinschätzung der Abgeordneten auch mit der Fremdwahrnehmung der Parlamentarier durch die Verwaltung übereinstimmt und ob sich die Beamten auf den jeweiligen Hierarchieebenen in der Einschätzung ihres Gegenübers unterscheiden. Werden verschiedene Rollenwahrnehmungen und Informationsasymmetrien zudem eher als funktional notwendige Arbeitsteilung begriffen, oder interpretieren die Akteure dies eher als Gegebenheit, die dem Vertrauen zwischen den Teileliten abträglich ist?

\section{Europäisierung von Fachpolitiken - Quell neuer Rollen in Parlament und Verwaltung?}

Dass in den vergangenen Jahrzehnten Gesetzgebungskompetenzen in großem Umfang auf die europäische Ebene verlagert wurden, gilt als eine wesentliche Ursache für die Entparlamentarisierung auf nationaler Ebene. Die nationale Vorbereitung der deutschen Europapolitik erfolgt im Wesentlichen in den Ministerien, nicht aber in den Arbeits- und Fachstrukturen des Parlaments. Die Beamten arbeiten die deutsche Position innerhalb der Ministerien - nach Art eines die ministerielle Hierarchie durchlaufenden Paternoster-Prinzips - aus; ein Teil von ihnen ist in die Arbeitsgruppen der EU-Kommission, des Rates sowie in die Komitologie eingebunden, angesichts des nicht gegebenen Verwaltungsunterbaus der EU aber auch - top down - an der Implementation europäischer Politik beteiligt. Gleichzeitig werden Beamte auch vorübergehend nach Brüssel entsandt. Der Bundestag und - noch wesentlich mehr - die Landtage sind demgegenüber vom europapolitischen Prozess stärker abgekoppelt und nicht institutionell in den Brüsseler Entscheidungsprozess eingebunden. Sieht man vom Europaausschuss des Bundestages oder zum Beispiel vom entsprechenden Unterausschuss des Haushaltsausschusses zu Fragen der Europäischen Union ab, ist „Europafähigkeit“ bisher in erster Linie ein Attribut der Exekutive geblieben auch wenn die Ministerialverwaltung durch transnationale Verwaltungsnetzwerke in manchen Politikfeldern ebenfalls an Einfluss verloren haben mag. ${ }^{77}$ Jedoch hat der Bundestag in den vergangenen Jahren seine Verfahren und die organisatorische Gliederung stärker an den Notwendigkeiten des europäisierten Politikzyklus ausgerichtet und dabei erkennbare Fortschritte erzielt. ${ }^{78}$

Nachholbedarf gegenüber der Exekutive besteht jedoch nicht nur hinsichtlich der institutionellen Anpassung, sondern auch bei der „Europafähigkeit“ des Personals. Die Beamten werden für ihren Einsatz in Brüssel, etwa mithilfe der Bundesakademie für öffentliche Ver-

77 Vgl. zur Debatte für viele Klaus H. Goetz, Europäisierung der öffentlichen Verwaltung - oder europäische Verwaltung?, in: Jörg Bogumil / Werner Jann / Frank Nullmeier (Hrsg.), a.a.O. (Fn. 2), S. $472-490$.

78 Vgl. Christian Demuth, Der Bundestag als lernende Institution. Eine evolutionstheoretische Analyse der Lern- und Anpassungsprozesse des Bundestages, insbesondere an die Europäische Integration, Baden-Baden 2009, S. 257 ff. 
waltung (BAköV), geschult. ${ }^{79}$ Vergleichbare, auf die Europäisierung zugeschnittene und das Rollenverständnis vielleicht in Richtung Europa transformierende Fortbildungen gibt es für die Abgeordneten und ihre Mitarbeiterstäbe kaum. Der EU-Ausschuss widmet sich vor allem integrationspolitischen Belangen; die Fachpolitiker der Fraktionen wiederum sehen „Europa“, obwohl es ihre alltägliche Arbeit unmittelbar berührt, eher als eine Aufgabe des EU-Ausschusses an. ${ }^{80}$ In den Fachausschüssen wurden EU-Politiken bislang wenig diskutiert, es sei denn, sie hatten eine gewisse politische Brisanz erreicht. So erklärt sich auch die noch immer dominierende Zurückhaltung des Bundestages, die im äußersten Fall dazu führte, dass die Abgeordneten, wie beim Europäischen Haftbefehl, einem nicht-verfassungskonformen Gesetz zustimmten. ${ }^{81}$ Erst in jüngerer Zeit bemühen sich die Fraktionen stärker darum, zum Beispiel durch informelle Vernetzungen mit europäischen Institutionen, schon in einem frühen Stadium auf den Entscheidungsprozess Einfluss zu nehmen. ${ }^{82}$

Angesichts der asymmetrischen Informationsverteilung und der ungleichgewichtigen Machtbeziehungen zwischen Legislative und Exekutive in europäisierten Politiken ist es wahrscheinlich, dass sich die wechselseitigen Rollenwahrnehmungen zwischen Ministerialbürokratie und Abgeordneten verändern. Schon wegen der enormen Arbeitsbelastung und der zeitlichen Restriktionen, die auf die enge Einbindung des Parlaments in nationale Gesetzgebungsprozesse sowie auf die ebenfalls aufwendige Wahlkreisarbeit zurückzuführen sind, bleiben vielen Fachpolitikern nur begrenzte Ressourcen, sich um europäisierte Fachpolitiken zu kümmern. Potentielle Rollenkonflikte, die sich durch das Spannungsverhältnis zwischen konkreten EU-Entscheidungen und parteipolitisch geprägten Policy-Positionen ergeben, werden bis heute weitgehend durch die grundsätzliche Befürwortung der europäischen Integration absorbiert. ${ }^{83}$ So sahen sich die Abgeordneten des Bundestages in den 1990er Jahren bezeichnenderweise als Statthalter des Europäischen Parlaments, bis dieses zu einem Vollparlament mit umfassenden Funktionen herangewachsen sei. ${ }^{84}$

Es liegt nahe, dass sich die Fachpolitiker der Fraktionen nicht den gleichen Expertenstatus zuschreiben, wie sie dies - den oben zitierten älteren Zahlen zufolge - ganz selbstver-

79 Vgl. Benedikt Speer, Europafähigkeit des öffentlichen Dienstes und Europakompetenz öffentlich Bediensteter. Anforderungen des Europäischen Verwaltungsraums an das deutsche System, in: Siegfried Magiera / Karl-Peter Sommermann / Jacques Ziller (Hrsg.), Verwaltungswissenschaft und Verwaltungspraxis in nationaler und transnationaler Perspektive, Berlin 2008, S. 683 - 703.

80 Vgl. Sabine Kropp, a.a.O. (Fn. 71).

81 In seinem Urteil zum Europäischen Haftbefehl hatte das Gericht die mangelhafte Kontrolle der Bundesregierung durch den Bundestag gerügt, vgl. BVerfGE, 2 BvR 2236/04 vom 18. Juli 2005.

82 Vgl. hierzu Bernhard Weßels, Roles and Orientations of Members of Parliament in the EU Context: Congruence or Difference? Europeanisation or Not?, in: Journal of Legislative Studies, 11. Jg. (2005), H. 3, S. 446 - 465. Weßels untersuchte unter anderem Kontakthäufigkeiten von Bundestagsabgeordneten zu europäischen Institutionen. Diese sagen indessen wenig darüber aus, wie die Bundestagsabgeordneten ihre eigenen Einflussmöglichkeiten einschätzen, ob sie Kontrollmöglichkeiten gegenüber der Exekutive geltend machen und ob sie sich gegenüber der Verwaltung als ebenbürtiger Rollenpartner betrachten. Rund 57 Prozent gaben der Studie zufolge an, immerhin mittelstark (38 Prozent stark) in den europäischen Policy-making-Prozess im eigenen Bereich eingebunden zu sein. In den von der Verfasserin durchgeführten qualitativen Interviews wird diese Beteiligung indessen vor allem durch die Fachpolitiker der Fraktionen skeptischer bewertet.

83 Vgl. Sabine Kropp, a.a.O. (Fn. 71).

84 Vgl. Richard S. Katz, Representation, the Locus of Democratic Legitimation and the Role of the National Parliaments in the European Union, in: ders. / Bernhard Weßels (Hrsg.), The European Parliament, the National Parliaments, and European Integration, Oxford 1999, S. 21 - 44. 
ständlich in der nationalen Arena tun. Zudem ist zu vermuten, dass die Fachpolitiker der Fraktionen von der Ministerialbürokratie, die älteren Studien zufolge der Politik (und damit den Abgeordneten) eine größere Kompetenz bescheinigte als allen anderen Teileliten, nicht in gleichem Maße als Experten akzeptiert werden, wie dies im Rahmen nationaler Gesetzgebung der Fall ist - und selbst dort sahen sie sich, wie oben gezeigt, im Rückstand gegenüber der Verwaltung. Zwar bezeichnete sich in einer Interviewstudie ein Teil der befragten Abgeordneten nachdrücklich als Fachpolitiker, der seinen Aktionsradius gezielt auf den EU-Politikzyklus ausdehnt und sich der Ministerialbürokratie durchaus ebenbürtig fühlt. Dies weist darauf hin, dass sich zögerlich eine neue Abgeordnetenrolle, nämlich die eines „europafähigen“ Fachpolitikers, ausbildet. ${ }^{85}$ Befördert wurde dies durch kritische Ereignisse wie den oben erwähnten Europäischen Haftbefehl sowie durch die Erfahrung, dass konkrete Entscheidungen der EU immer wieder mit parteipolitisch geprägten Politikpositionen kollidieren. Auch die Fraktionsvorstände, etwa der CDU/CSU, halten mittlerweile einige ihrer Sitzungen in Brüssel ab, um schon in einem frühen Stadium des EU-Politikzyklus politisch relevante Informationen einzuholen und auf Entscheidungsprozesse Einfluss nehmen zu können. Geht man, dem neo-institutionalistischen Paradigma folgend, ferner davon aus, dass Institutionen Anreize für Akteurstrategien setzen, scheint es zumindest nicht ausgeschlossen, dass das Urteil des Bundesverfassungsgerichts zum Lissabon-Ver$\operatorname{trag}^{86}$, das eine stärkere „Integrationsverantwortung " des Bundestages anmahnte, sowie das damit einhergehende Begleitgesetz des Bundestages eine stärkere Hinwendung der Abgeordneten zu europäisierten Politiken bewirken. ${ }^{87}$

Beide Annahmen bedürfen allerdings einer Überprüfung auf der Grundlage stärker repräsentativer Erhebungen. Die bisherigen Befunde weisen jedenfalls auf einen zaghaften Wandel im Rollenverständnis der Abgeordneten hin, der bislang aber durch die grundsätzlich befürwortende Haltung der Eliten gegenüber der europäischen Integration eingehegt worden ist. Europaskeptische Positionen, die vermutlich zu einer stärkeren Kontrolltätigkeit des Parlaments führen, waren bisher im Bundestag kaum vertreten. Eine Ausnahme von der Regel stellt derzeit allenfalls die Fraktion der Linkspartei dar ${ }^{88}$, die dem LissabonVertrag nicht zustimmte und letztlich gegen ihn klagte. Befürworter dieses Vertrags wurden zudem nicht wieder als Kandidaten für die Europawahlen 2009 aufgestellt. Es ist somit anzunehmen, dass sich auch parteipolitisch divergierende „europäisierte“ Abgeordnetenrollen im Bundestag aufspüren lassen. Ob stärker europaskeptische Positionen tatsächlich auch mit einer intensiveren Kontrolltätigkeit einhergehen, muss sich erst erweisen. Sie sind keineswegs eine hinreichende Bedingung dafür. Auch Sprachkenntnisse und soziale Techniken, die eine informelle Vernetzung mit Akteuren und Institutionen auf EU-Ebene erlau-

85 Vgl. im Folgenden Sabine Kropp, a.a.O. (Fn. 71).

86 BVerfGE, 2 BvE 2/08 vom 30. Juni 2009.

$87 \mathrm{Vgl}$. Roland Lhotta / Jörg Ketelhut, Integrationsverantwortung und parlamentarische Demokratie: Das Bundesverfassungsgericht als Agent des „verfassten politischen Primärraums“, in: ZParl, 40. Jg. (2009), H. 4, S. 846 - 888; Andreas Wimmel, Neue (alte) Konfliktlinien in der Europapolitik: Die Parlamentsdebatte zum Vertrag von Lissabon im Deutschen Bundestag, in: ZParl, 40. Jg. (2009), H. 4, S. 476 - 758; Sven Hölscheidt / Steffi Menzenbach / Birgit Schröder, Das Integrationsverantwortungsgesetz - ein Kurzkommentar, in: ZParl, 40. Jg. (2009), H. 4, S. 758 - 773.

88 Vgl. Charles Lees, The Limits of Party-Based Euroscepticism in Germany, in: Aleks Szczerbiak I Paul Taggart (Hrsg.), Opposing Europe? The Comparative Party Politics of Euroscepticism, Bd. 1: Case Studies and Country Surveys, Oxford 2008, S. $16-37$. 
ben, sowie entsprechende Aktivitäten der Fraktionen beziehungsweise Fraktionsführungen zählen zu den entsprechenden Voraussetzungen.

Gleichzeitig dürfte durch die Europäisierung des Bundestages auch die Zahl der Präferenzrollen im Parlament beziehungsweise in den Fraktionen zunehmen. Nun könnte man davon ausgehen, dass das Fraktionsmanagement dadurch auf größere Schwierigkeiten stoßen könnte, eine in sich konsistente Position nach außen zu vertreten - eine nicht unwesentliche Bedingung für die Berechenbarkeit und Vertrauenswürdigkeit eines kollektiven Akteurs. Erste Befunde weisen indessen darauf hin, dass die Abgeordneten selbst eine gewachsene Rollenvielfalt nicht als eine potentielle Beeinträchtigung der Handlungsfähigkeit betrachten. Vielmehr wird die weitere Ausdifferenzierung von Rollen als eine notwendige Voraussetzung für die Organisation Parlament beziehungsweise Fraktion erachtet, sich an eine komplexer werdende Umwelt anzupassen. ${ }^{89}$ Noch unbeantwortet ist jedoch, wie Ministerialbürokratie und Regierungsmitglieder diese Entwicklung bewerten.

Im nationalen Rahmen stellt die Ministerialbürokratie eine der bedeutendsten Informationsquellen für die Abgeordneten dar; sie ist zudem ein wichtiger Rollenpartner im Regierungsalltag. Ist dies auch der Fall, wenn europäisierte Fachpolitiken behandelt werden? Angesichts der bisherigen Zurückhaltung der Bundestagsabgeordneten bei Kontrolle und Mitsteuerung ist davon auszugehen, dass der Verwaltung als Interaktionspartner in europäisierten Fachpolitiken eine geringere Bedeutung zukommt als im nationalen Rahmen. In der Tat konnten die meisten befragten Abgeordneten die Beamten, die in die Arbeitsgruppen der Kommission und des Rates eingebunden sind, nicht identifizieren. Doch selbst die Fachpolitiker, die angaben, diese Rolle auf europäisierte Politiken auszudehnen, betonten, dass die Informationen, die sie zum Beispiel durch die Kontakte mit EP-Abgeordneten des gleichen parteipolitischen Lagers oder über Nichtregierungsorganisationen (NGOs) erhielten, besser verwertbar seien als die, die sie über die Ministerialbürokratie abfragen könnten. NGOs böten aufgrund der Tatsache, dass sie in europäische Dachverbände eingebunden und in Brüssel präsent sind, relevante Informationen nicht nur bereits im Prozess des Agenda-Settings, sondern eröffneten darüber hinaus eine vergleichende Sicht, indem sie die Positionen anderer Mitgliedstaaten und Akteure in ihr Handeln einbezögen. Auch bewerteten diese Abgeordneten ihre europapolitische Aktivität als erforderlich, um die mitunter ,abgehobenen" Entscheidungen der EU stärker mit einer bürgernahen Perspektive zu verbinden. Sie begründeten somit die Europäisierung der Fachpolitikerrolle mit einer Repräsentationsperspektive, die auf das „Gemeinwohl“ bezogen ist und durch die Institutionen der EU und ihre Repräsentanten nicht hinreichend abgedeckt werden könne. ${ }^{90}$ Dies könnte auf ein verändertes Repräsentationsverständnis zumindest einzelner Abgeordneter des Bundestages hinweisen, die dessen Funktion als „Statthalter" für das noch nicht zum EP herangereifte Europäische Parlament nicht mehr uneingeschränkt bejahen. Inwiefern man bereits von einem Wandel sprechen kann, der die bei europäisierten Fachpolitiken bisher offenbar weitgehend anerkannte Rollenteilung zwischen Exekutive und nationalem Parlament durchkreuzt, kann noch nicht sicher beantwortet werden.

Andere Abgeordnete wiederum griffen, um das Verhältnis zwischen Exekutive und Parlament in europäisierten Fachpolitiken erläutern zu können, auf eine Uminterpretation des Gewaltenteilungsgrundsatzes zurück: Da die Beteiligung an Entscheidungen der EU Sache

89 Vgl. Sabine Kropp, a.a.O. (Fn. 71).

90 Vgl. ebenda. 
der Regierungen und Verwaltungen sei, existiere eine Art natürlicher Vorrang der Exekutive, mithin ein exekutivischer Kernbereich, in den die Abgeordneten nicht eingreifen sollten. Täten sie dies, würde der ohnehin häufig beklagte vielstimmige Chor deutscher Positionen in Brüssel nur noch mehr Dissonanzen hervorbringen. Dass sich die Abgeordneten während der Ausarbeitung von EU-Politiken weitgehend auf die Expertise der Exekutive verlassen und während laufender Verhandlungen nur selten intervenieren, betrachten insbesondere die Parlamentarier der Mehrheitsfraktionen als Ausdruck eines stabilen Vertrauens zwischen Parlament, Regierung und Verwaltung. Der Sachverstand liegt ihren Aussagen zufolge eindeutig auf der Seite von Regierung und Verwaltung, der folgerichtig die Expertenrolle zuerkannt wird, die sie sich auch selbst zuschreibt. Dieses Vertrauen schließe aber eine Kontrolle durch die Parlamentarier grundsätzlich nicht aus. ${ }^{91}$ Jedoch zeigt die bisherige Erfahrung, dass die Kontrolltätigkeit hinter das dominierende Vertrauen zurücktritt. Angesichts der oben referierten Befunde ist aber zu vermuten, dass sich der Kontrollaspekt mit dem Wechsel von einer Großen Koalition zum stärker bipolaren Wechselspiel von „kleiner“ Regierungsmehrheit und Opposition nach den Wahlen von 2009 wieder stärker ausbildet und gegebenenfalls auch das Handeln von Regierung und Verwaltung auf europäischer Ebene mehr als bislang einschließt.

Dem Idealbild, dass jeder Parlamentarier die europäische Dimension seines Handelns stets mit bedenken solle, entspricht die Realität derzeit jedenfalls nicht. Dies vermag insofern kaum zu verwundern, als sich die Abgeordneten nach der auch die formalen Positionsrollen definierenden Logik des Arbeitsparlaments verhalten: Sie wirken zuvorderst an den Materien mit, die sie beeinflussen können. Bisherige, vorläufige Befunde deuten darauf hin, dass die „Wahlkreisarbeiter“ ihrer Rolle weniger eine europäisierte Dimension beifügen als die „Fachpolitiker“ (und, erst recht, der Europapolitiker der Fraktionen). Sie verweisen zudem eher auf die oben genannte Heuristik des Gewaltenteilungsgrundsatzes, um eine Rollenteilung zwischen Parlament und Verwaltung zu begründen. Die „Parlamentsmanager“, die Führungspositionen im Bundestag oder in den Fraktionen innehaben und Steuerungsaufgaben übernehmen, sind offensichtlich wiederum stärker mit den Institutionen der EU vernetzt. ${ }^{92}$ Offenbar hängt der Grad der Europäisierung bei den Abgeordneten nicht zuletzt davon ab, welche der oben erwähnten Repräsentations-, Positions- und Präferenzrollen sie verinnerlicht haben: Diese Hypothese wäre jedoch auf der Grundlage einer repräsentativen Fallzahl weiter zu überprüfen.

Über die gegenläufige Perspektive, die „europäisierte“ Rollenwahrnehmung der deutschen Ministerialbeamten auf den verschiedenen Hierarchiestufen, ist wenig bekannt. Die letzten Verwaltungsstudien haben diesen Aspekt nicht berücksichtigt. Aus vergleichenden Untersuchungen, etwa zur schwedischen, norwegischen oder niederländischen Bürokratie $^{93}$, wissen wir, dass nach Brüssel entsandte oder in die Arbeitsgruppen der Kommission

91 Vgl. ebenda.

92 Vgl. ebenda.

93 Vgl. für viele Jarle Trondal / Frode Veggeland, Access, Voice and Loyalty: the Representation of Domestic Civil Servants in EU Committees, in: Journal of European Public Policy, 10. Jg. (2003), H. 1, S. 59 - 77; Jarle Trondal, Is the European Commission a "Hothouse" for Supranationalism? Exploring Actor-Level Supranationalism, in: Journal of Common Market Studies, 45. Jg. (2007), H. 5, S. 1111 - 1133; Morten Egeberg, Executive Politics as Usual Role Behaviour and Conflict Dimensions in the College of European Commissioners, in: Journal of European Public Policy, 13. Jg. (2006), H. 1, S. 1 - 15. Vgl. auch Karin Geuijen / Paul t'Hart / Sebastiaan Princen / 
oder des Rates eingebundene nationale Beamte ihr Rollenverständnis stärker an den Legitimations- und Regulierungsbedürfnissen der europäischen Ebene ausrichten. Sie sind als Promotoren nationaler und europäischer Interessen zudem mehr als die Beamten, die ausschließlich im nationalen Kontext wirken, Rollenkonflikten ausgesetzt. Aus der oben zitierten Interviewstudie geht hervor, dass die Bundestagsabgeordneten das Auftreten der deutschen Kommissionsbeamten gegenüber dem nationalen Parlament als abgehoben empfinden. Das Verhältnis zwischen den EU-Beamten und den Abgeordneten scheint weitgehend von wechselseitiger Ignoranz geprägt; nach Angaben befragter Parlamentarier sei dies in anderen EU-Mitgliedstaaten anders. ${ }^{94}$ Noch weniger ist darüber bekannt, welche Rollenerwartungen die an europäisierten Fachpolitiken mitwirkenden nationalen Beamten sowie die „Eurokraten“, also die nach Brüssel entsandten deutschen EU-Beamten, an die Abgeordneten richten, und ob diese Erwartungen wiederum dem eigenen Rollenverständnis der Abgeordneten entsprechen. Es spricht jedoch einiges dafür, dass die Vertrauensbeziehungen zwischen den Teileliten von Parlament und Verwaltung anders und komplexer gelagert sind, sobald sie die europäische Dimension des Policy-Making einbeziehen. Das Verhältnis zwischen den Fachpolitikern der Fraktionen und den Beamten dürfte insgesamt asymmetrischer ausfallen als im nationalen Rahmen, denn das Gros der Abgeordneten hat sich bei der Politikformulierung in der Vergangenheit eher zurückhaltend gezeigt.

Beide Gruppen sind in unterschiedlicher Intensität in die Mehrebenenpolitik der EU eingebunden. Dies ruft mit einer gewissen Wahrscheinlichkeit Rollenkonflikte hervor, die bislang aber kaum aufgebrochen sind. Bisherigen Erkenntnissen zufolge lösen die Abgeordneten solche potentiellen Konflikte häufig über Urteilsheuristiken auf, die ihrerseits komplexe Handlungssituationen vereinfachen und nachvollziehbare "Regieanweisungen " anbieten. ${ }^{95}$ Auch in dieser Hinsicht besteht Forschungsbedarf in der Parlaments- und Verwaltungsforschung, und zwar sowohl in konzeptioneller als auch in empirischer Hinsicht.

\section{Rollen in Parlament und Verwaltung - eine Forschungsagenda}

Die Handlungsfähigkeit von Politik hängt - dies zeigt auch die vergleichende politikwissenschaftliche Forschung - wesentlich davon ab, ob das Verhältnis zwischen den Eliten des politisch-administrativen Systems von Vertrauen getragen ist oder nicht. Deshalb ist es von erheblichem Interesse, inwieweit die Rollen, die sich Politik und Verwaltung selbst zuweisen, kompatibel sind, inwieweit Selbst- und Fremdwahrnehmung übereinstimmen und ob sich beide Gruppen aus der Sicht des jeweiligen Gegenübers so verhalten, wie es der Rollenzuschreibung durch die jeweils andere Gruppe entspricht. Verwaltungs- und Parlamentsforschung teilen somit ein ähnliches Forschungsinteresse, auch wenn die Vertreter beider Zweige sowohl im nationalen Kontext als auch in international vergleichenden Arbeitszusammenhängen bislang kaum beziehungsweise allenfalls im Rahmen von Elitestudien kooperieren - ein Desiderat, für dessen Behebung dieser Beitrag mit Nachdruck plädiert.

Kutsal Yesilkagit, a.a.O. (Fn. 10); Jarle Trondal / Caspar van den Berg / Semin Suvarierol, The Compound Machinery of Government: The Case of Seconded Officials in the European Commission, in: Governance, 21. Jg. (2008), H. 2, S. $253-274$.

94 Vgl. Sabine Kropp, a.a.O. (Fn. 71).

95 Vgl. Richard R. Lau, Models of Decision-Making, in: David O. Sears / Leonie Huddy / Robert Jervis (Hrsg.), Oxford Handbook of Political Psychology, Oxford 2003, S. 19 - 59. 


\begin{tabular}{|c|c|c|c|}
\hline \multicolumn{4}{|c|}{$\begin{array}{l}\text { Tabelle 1: Verwaltung und Parlament als Rollenpartner - Forschungsstand und Forschungs- } \\
\text { desiderate }\end{array}$} \\
\hline $\begin{array}{l}\text { Untersuchungs- } \\
\text { dimension }\end{array}$ & $\begin{array}{l}\text { Verwaltungs- } \\
\text { wissenschaft }\end{array}$ & $\begin{array}{l}\text { Parlaments- } \\
\text { forschung }\end{array}$ & $\begin{array}{l}\text { Studien mit ,inte- } \\
\text { grierter“ Perspektive }\end{array}$ \\
\hline $\begin{array}{l}\text { Eigenes Rollenver- } \\
\text { ständnis Verwaltung/ } \\
\text { Parlament }\end{array}$ & $\begin{array}{l}\text { Mehrfach replizierte } \\
\text { Studien zum Rollenbild } \\
\text { der Verwaltung }\end{array}$ & $\begin{array}{c}\text { Ausdifferenzierte } \\
\text { Literaturlage zu } \\
\text { Abgeordnetenrollen }\end{array}$ & Einige ältere Studien \\
\hline $\begin{array}{l}\text { Rollenquervergleich, } \\
\text { wechselseitige } \\
\text { Perzeptionen }\end{array}$ & $\begin{array}{l}\text { Einige Ansatzpunkte } \\
\text { in älteren Studien, } \\
\text { in neueren Studien aber } \\
\text { kaum enthalten }\end{array}$ & $\begin{array}{l}\text { Einige wenige Aus- } \\
\text { sagen, keine expliziten } \\
\text { Untersuchungen; } \\
\text { neuere Studien mit } \\
\text { explorativem } \\
\text { Charakter }\end{array}$ & $\begin{array}{l}\text { Einzelne Ansatzpunkte } \\
\text { vor allem in der Eliten- } \\
\text { forschung und älteren } \\
\text { Verwaltungsforschung } \\
\text { (und in der Parlaments- } \\
\text { forschung) }\end{array}$ \\
\hline $\begin{array}{l}\text { Europäisierung } \\
\text { des Rollenverständ- } \\
\text { nisses }\end{array}$ & $\begin{array}{l}\text { Wenige Anhaltspunkte zu } \\
\text { Deutschland; Informatio- } \\
\text { nen eher in international } \\
\text { vergleichenden Studien, } \\
\text { vor allem zu Skandinavien } \\
\text { und den Niederlanden }\end{array}$ & $\begin{array}{l}\text { Einzelne Aspekte in } \\
\text { Studien, dabei aber } \\
\text { kaum Aussagen zu } \\
\text { Vernetzungen zwischen } \\
\text { Parlament und Verwal- } \\
\text { tung }\end{array}$ & $\begin{array}{c}\text { Nur explorative } \\
\text { Studien zu wechsel- } \\
\text { seitigen Perzeptionen } \\
\text { in europäisierten } \\
\text { Fachpolitiken }\end{array}$ \\
\hline $\begin{array}{l}\text { Rollen nach Hierar- } \\
\text { chieebenen in der } \\
\text { Organisation „Parla- } \\
\text { ment“ beziehungs- } \\
\text { weise „Verwaltung“ }\end{array}$ & $\begin{array}{l}\text { Nur wenige Informatio- } \\
\text { nen; bisherige Studien vor } \\
\text { allem bezogen auf das } \\
\text { Führungspersonal der } \\
\text { öffentlichen Verwaltung }\end{array}$ & $\begin{array}{l}\text { Parlamentsmanager } \\
\text { versus Hinterbänkler }\end{array}$ & nein \\
\hline $\begin{array}{l}\text { Mehrebenenvergleich } \\
\text { von Rollen }\end{array}$ & nein & $\begin{array}{c}\text { Einzelne Aussagen in } \\
\text { neueren repräsentativen } \\
\text { Parlamentsstudien } \\
\end{array}$ & nein \\
\hline $\begin{array}{l}\text { Regionale Unter- } \\
\text { schiede (Ost-/West- } \\
\text { deutschland) }\end{array}$ & $\begin{array}{l}\text { Veraltete Daten, daher kein } \\
\text { Aufschluss über eventuelle } \\
\text { Pfadabhängigkeiten }\end{array}$ & $\begin{array}{l}\text { Neuere repräsentative } \\
\text { Parlamentsstudien }\end{array}$ & nein \\
\hline $\begin{array}{l}\text { Verwaltungskultur - } \\
\text { Auswirkungen des } \\
\text { New Public Manage- } \\
\text { ment (NPM) bezie- } \\
\text { hungsweise „,neo- } \\
\text { weberianischen } \\
\text { Staates“ }\end{array}$ & $\begin{array}{l}\text { Keine Untersuchungen, } \\
\text { die Aufschluss über verän- } \\
\text { dertes Rollenverständnis in } \\
\text { der Ministerialbürokratie } \\
\text { geben, nur einzelne } \\
\text { Anhaltspunkte }\end{array}$ & - & nein \\
\hline $\begin{array}{l}\text { Unterschiede } \\
\text { Opposition versus } \\
\text { Regierungsmehrheit }\end{array}$ & $\begin{array}{l}\text { Vereinzelte Aussagen } \\
\text { über die Affinität von } \\
\text { Beamten zu Regierung } \\
\text { und Opposition }\end{array}$ & $\begin{array}{l}\text { Aussagen über die } \\
\text { Verwaltung als Rollen- } \\
\text { partner in älteren } \\
\text { Studien }\end{array}$ & nein \\
\hline
\end{tabular}

Tabelle 1 fasst den Forschungsstand im Überblick zusammen. Teilt man die verschiedenen, oben erläuterten Untersuchungsdimensionen des Forschungsgebiets in Forschungszweige ein - also in Verwaltungswissenschaft und Parlamentsforschung - und fügt dieser Unterscheidung ferner die in diesem Beitrag als besonders bedeutend eingestufte wechselseitige Wahrnehmung und Rollenzuschreibung als analytischen Bezugspunkt hinzu, erkennt man sofort, dass insbesondere bei den Studien mit „integrierter" Perspektive noch erhebliche 
Forschungslücken klaffen. Politik- und Verwaltungswissenschaft verfügen nur über wenige Anhaltspunkte und Ergebnisse zu wechselseitigen Rollenzuschreibungen von Abgeordneten, Regierungsmitgliedern und Verwaltung. Es fällt zudem auf, dass insbesondere die neuere Verwaltungsforschung insofern „parlamentsblind“ agiert, als Abgeordnete als Rollenpartner in Untersuchungen kaum berücksichtigt werden, obwohl es in der Realität vielfache Interaktionsbeziehungen gibt. Demgegenüber enthalten Abgeordnetenstudien einzelne Aussagen zur Verwaltung als Interaktionspartner des Parlaments, auch wenn sie Rollen der Ministerialbürokratie aufgrund ihres klar umrissenen Forschungsschwerpunkts und wegen zumeist begrenzter Mittel weder theoretisch integrieren noch empirisch explizit mit erheben. Grundsätzlich gilt jedoch: Der Rollenquervergleich ist bislang systematisch kaum angestellt worden; Anhaltspunkte finden sich ehestens in inzwischen älteren Elitestudien.

Noch spärlicher fallen gesicherte Erkenntnisse darüber aus, wie sich die jeweils eigene und ebenso die wechselseitige Rollenzuschreibung von Parlamentariern und Verwaltung durch die Europäisierung von Fachpolitiken und durch die vertiefte europäische Integration verändert haben. Gerade dieser Aspekt hat jedoch die Asymmetrie zwischen Exekutive und Legislative nicht unwesentlich verstärkt. Er ist für die Forschungen über die Steuerungsund Implementationskreisläufe in der europäischen Multilevel Governance zudem von großer Bedeutung. Kann man angesichts der oben referierten Befunde noch davon ausgehen, dass die Rollen beider Gruppen, Expertenrollen wie Repräsentationsrollen, mit gewissen Abstrichen im Rahmen nationaler Politiken wechselseitig anschlussfähig sind, so ist dies im Zuge der Europäisierung nicht unbedingt der Fall. Die wechselseitige Zuschreibung der Expertenrolle, die für Abgeordnete und Beamte typisch ist, dürfte davon nicht unberührt geblieben sein. Unklar ist ebenfalls, ob und wie die zögerliche, auch vom Bundesverfassungsgericht angemahnte stärkere Einbindung des Bundestages in EU-Politiken seitens der Exekutive bewertet und wie sie künftig in konkrete Rollenerwartungen an die Abgeordneten übersetzt wird. Auch bedarf die festgestellte Ausbildung einer europäisierten Fachabgeordnetenrolle einer weiteren Untersuchung in repräsentativen Erhebungen. Die Forschung kann somit derzeit nur auf einzelne Anhaltspunkte zurückgreifen, wie sich die Europäisierung auf das für den nationalen Handlungsrahmen so akribisch herausgearbeitete Rollenset der Abgeordneten auswirkt ${ }^{96}$; dasselbe lässt sich über die Verwaltungsforschung und die Beamten sagen. Sieht man von der kommunalen Ebene ab, hat jene ferner - trotz einer jahrzehntelang währenden Debatte über das sogenannte New Public Management - kaum systematische Ergebnisse hervorgebracht, ob und wie sich diese Debatte im Rollenverständnis der Ministerialbürokratie niederschlägt. Auch die Erkenntnisse, die nach dem Vollzug der deutschen Einheit über unterschiedliche Verwaltungskulturen in West- und Ostdeutschland zutage gefördert wurden, sind mittlerweile veraltet und lassen kaum Aussagen über mögliche, langfristig wirksame Pfadabhängigkeiten oder über Anpassungsprozesse zu.

In einem Forschungszusammenhang lassen sich die in diesem Beitrag skizzierten Fragestellungen allerdings nicht bearbeiten. Sie beschreiben eher ein Forschungsprogramm, das aufzunehmen sich angesichts der bestehenden Forschungslücken, der Relevanz und Anschlussfähigkeit eines solchen Vorhabens gegenüber weiteren Forschungszweigen jedoch lohnen dürfte.

96 Vgl. Christian Demuth, a.a.O. (Fn. 78). 